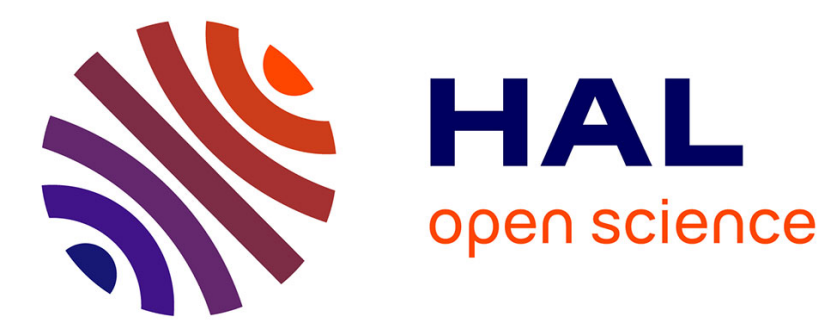

\title{
Transient growth in the flow past a three-dimensional smooth roughness element
}

Stefania Cherubini, Marco de Tullio, Pietro de Palma, Giuseppe Pascazio

\section{To cite this version:}

Stefania Cherubini, Marco de Tullio, Pietro de Palma, Giuseppe Pascazio. Transient growth in the flow past a three-dimensional smooth roughness element. Journal of Fluid Mechanics, 2013, 724, pp.642-670. 10.1017/jfm.2013.177 . hal-01086742

\section{HAL Id: hal-01086742 https://hal.science/hal-01086742}

Submitted on 24 Nov 2014

HAL is a multi-disciplinary open access archive for the deposit and dissemination of scientific research documents, whether they are published or not. The documents may come from teaching and research institutions in France or abroad, or from public or private research centers.
L'archive ouverte pluridisciplinaire HAL, est destinée au dépôt et à la diffusion de documents scientifiques de niveau recherche, publiés ou non, émanant des établissements d'enseignement et de recherche français ou étrangers, des laboratoires publics ou privés. 


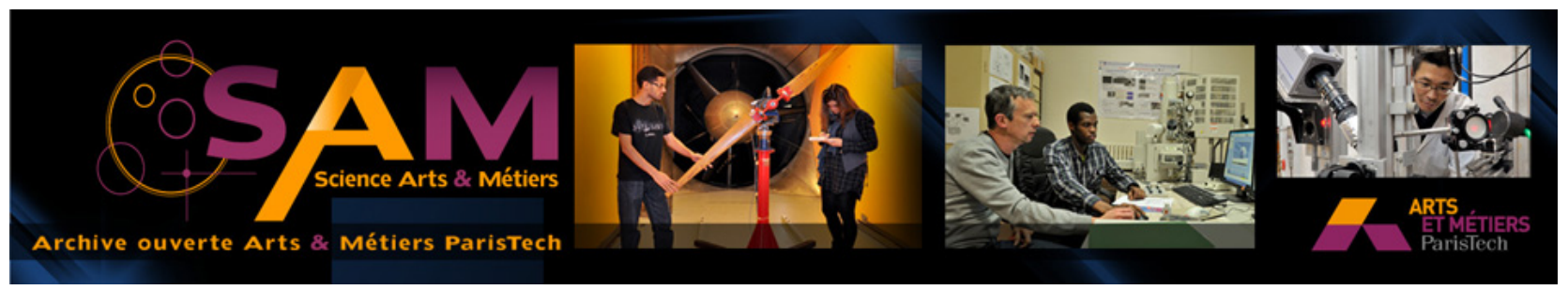

Science Arts \& Métiers (SAM)

is an open access repository that collects the work of Arts et Métiers ParisTech researchers and makes it freely available over the web where possible.

This is an author-deposited version published in: http://sam.ensam.eu

Handle ID: .http://hdl.handle.net/10985/8973

\section{To cite this version :}

Stefania CHERUBINI, Marco DE TULLIO, Pietro DE PALMA, Giuseppe PASCAZIO - Transient growth in the flow past a three-dimensional smooth roughness element - Journal of Fluid Mechanics - Vol. 724, p.642-670. - 2013 


\title{
Transient growth in the flow past a three-dimensional smooth roughness element
}

\author{
S. CHERUBIN I I,2, M. D. DE TULLIO ${ }^{1}$, P. DE PAL MA ${ }^{1}$, \\ and G. P A S C A Z I O ${ }^{1}$ \\ ${ }^{1}$ DIMeG, CEMeC, Politecnico di Bari, Via Re David 200, 70125 Bari, Italy \\ ${ }^{2}$ DynFluid, Arts et Metiers ParisTech, 151, Bd. de l'Hopital, 75013 Paris, France
}

(Received ?? and in revised form ??)

This work provides a global optimization analysis, looking for perturbations inducing the largest energy growth at a finite time in a boundary-layer flow in the presence of smooth three-dimensional roughness elements. Amplification mechanisms are described which can by-pass the asymptotical growth of Tollmien-Schlichting waves. Smooth axisymmetric roughness elements of different height have been studied, at different Reynolds numbers. The results show that even very small roughness elements, inducing only a weak deformation of the base flow, can localize the optimal disturbance characterizing the Blasius boundary-layer flow. Moreover, for large enough bump heights and Reynolds numbers, a strong amplification mechanism has been recovered, inducing an increase of several order of magnitude of the energy gain with respect to the Blasius case. In particular, the highest value of the energy gain is obtained for an initial varicose perturbation, differently to what found for a streaky parallel flow. Optimal varicose perturbations grow very rapidly by transporting the strong wall-normal shear of the base flow, which is localized in the wake of the bump. Such optimal disturbances are found to lead to transition for initial energies and amplitudes considerably smaller than sinuous optimal ones, inducing hairpin vortices downstream of the roughness element.

\section{Introduction}

Transition to turbulence in spatially developing boundary-layer flows is a fundamental issue for many aerodynamic and industrial applications, but its understanding, prediction and control still remain a challenging issue. For small amplitude disturbances and supercritical Reynolds numbers, the linear stability analysis predicts the slow transition process due to the generation, amplification and secondary instability of Tollmien-Schlichting (TS) waves (Schmid \& Henningson 2001). However, even if the regime is subcritical with respect to TS waves (i.e., all eigenmodes are damped), a so-called by-pass transition to turbulence is often observed. In fact, due to the non-normality of the Navier-Stokes operator, disturbances could experience a large transient amplification, arising from the constructive interference of damped non-orthogonal eigenmodes (Schmid \& Henningson 2001). If growth is sufficient, such amplified structures could induce secondary instability and breakdown, leading to a by-pass transition (see Schoppa \& Hussain (2002); Brandt et al. (2004)).

With the aim of analysing the early phases of bypass transition, the concept of "optimal perturbation" was introduced (see Farrell (1988); Butler \& Farrell (1992)), which is defined as the perturbation of the steady base flow inducing the maximum energy growth at a target time. Since then, many works have focused on the "local" stability 
analysis of parallel and weakly non-parallel flows, looking for "local optimal perturbations" characterized by a given wavenumber in the streamwise and spanwise directions (Luchini 2000; Schmid 2000; Corbett \& Bottaro 2000). In a boundary layer, the local optimal perturbation is characterized by a streamwise-invariant counter-rotating vortex pair, and resulting at finite time in a pair of streamwise velocity streaks. The results of such an analysis have been very promising, being able to predict the onset of elongated structures which are indeed observed in transitional boundary-layer flows (see Schoppa \& Hussain (2002); Brandt et al. (2004)). However, the drawbacks of such "local" methods are that they focus onto a single wavenumber/frequency and that they neglect the effects due to the non-parallelism of the flow. Thus, they appear to be not suitable to study the instability behaviour of non-parallel flows.

In the past few years, these limitations have been overtaken thanks to the development of the "global" stability analysis, which considers two (or three) eigendirections, allowing to study a non-parallel base flow with general perturbations, not characterized by a single wavenumber. For instance, such methods have been used to study the eigenmodes of a periodic wake of a circular cylinder (Barkley \& Henderson 1996); the convective and absolute instability of the flow in a curved channel (Marquet et al. 2008) and past a backward-facing step (Barkley et al. 2002; Blackburn et al. 2008); the asymptotical centrifugal instability and the non-normal growth in a recirculation bubble over a flat plate (Theofilis et al. 2000; Gallaire et al. 2007; Cherubini et al. 2010 c). A review of the global instability methods and their applications is provided in Theofilis (2003). Such methods are very powerful since, even in weakly non-parallel flows, they allow to take into account the transient amplification of the disturbance energy due to the non-orthogonality of the eigenvectors, providing a useful tool to study the early phases of by-pass transition. For instance, in the case of the boundary-layer flow, a global instability analysis has recently assessed that streamwise-modulated counter-rotating vortices are transiently amplified more than streamwise-independent ones (see Cherubini et al. (2010b)). Such global optimal disturbances, modulated in the streamwise and spanwise direction, have been found to induce transition at lower energy than the streamwise-elongated local ones. This points out the importance of using global methods to study non-parallel and even weakly-non parallel flows such as the boundary-layer flow.

It is well-known that transition in a boundary-layer flow is triggered by exogenous disturbances, namely wall roughness, acoustic waves, or freestream turbulence. Although progress has been made in the study of the early phases of transition, the process by which surface roughness affects the laminar-turbulent transition is only partially understood. Pioneer work in this field has been performed experimentally by Gregory \& Walker (1956); Tani et al. (1962); Acalar \& Smith (1987), and numerically by Joslin \& Grosch (1995), for different roughness shapes (cones, cylinders, hemispheres, and bumps, respectively). All of this studies have identified a vortex-generation mechanism inducing streaks downstream of the roughness element, which causes strong distortions on the mean flow, modifying the asymptotical and transient dynamics of the flow. Since then, many experimental and numerical works have focused on cylindrical-shaped roughness elements (Tani et al. (1962); White (2002); White \& Ergin (2003); Fransson et al. (2004); Choudhari \& Fischer (2005) among the others), observing that, for such non-smooth roughness elements, bypass transition is reached at a critical value of the roughness Reynolds number, $R e_{k}$ (based on the height of the roughness and the Blasius boundary-layer streamwise velocity on top of the roughness element), indicating that a global modal instability may set in at such a critical value of $R e_{k}$. On the other hand, for subcritical values of $R e_{k}$, bypass transition is never observed, and a stabilizing effect of the roughness-induced streaks on the growth of TS waves has been found (see Fransson et al. (2004, 2005, 2006)). How- 
ever, all of these experimental studies have focused on arrays of non-smooth roughness elements having a non-small aspect ratio (between $1: 2$ and $1: 3$, height over diameter of the roughness element). Whereas, smaller efforts have been dedicated to shallow smooth roughness elements (bumps), which can be comparable to defects or imperfections of a flat plate. Indeed, shallow roughness elements having a smoother shape are expected to have a more subtle influence on bypass transition, since they induce weaker distortions of the mean flow than in the non-smooth case (Piot et al. 2008). Thus, it may be expected that smooth roughness elements will enhance the receptivity of the boundary-layer flow with respect to external disturbances, inducing transition to turbulence by means of a non-modal mechanism.

The first theoretical, experimental and numerical studies of the flow around isolated three-dimensional bumps (see Lipatov \& Vinogradov (2000); Joslin \& Grosch (1995)), have shown the existence of a complex pattern of streamwise streaks and vortices, similar to the optimal disturbances typical of a boundary-layer over a flat plate. More recently, Piot et al. (2008) have assessed the stabilizing nature of such roughness-induced streaks on the growth of TS waves. They have assumed that the flow past the smooth roughness element evolves slowly in the streamwise direction. Then, the local stability of the pre-streaky flow just behind the roughness element has been studied by considering the two-dimensional flow at each streamwise location. Solving an eigenvalue problem for each of the two-dimensional base flows obtained, the authors found that the modes have smaller growth rates then the associated Blasius TS-waves, assessing the stabilizing effect of the pre-streaky flow on the growth of TS waves. Nevertheless, the same authors observed, by using direct numerical simulations (DNS), that the interaction of such TS modes could induce an amplification of perturbation energy much larger than in the Blasius case. This behaviour could be due to the non-normality of the considered modes, leading to a transient amplification of the disturbance energy.

The aim of the present work is to assess whether smooth three-dimensional roughness elements are capable of inducing linear amplification mechanisms which by-pass the asymptotical growth of TS waves and lead the flow to transition even if all eigenmodes are stable. To this purpose, we perform a fully three-dimensional energy optimization analysis, looking for perturbations inducing the largest energy growth at a finite time in a boundary-layer flow in the presence of a three-dimensional bump. The shape of the roughness element has been described by the immersed boundary (IB) technique, useful to handle complex geometries, already employed for the stability analysis of the twodimensional flow past a cylinder by Giannetti \& Luchini (2007).

The paper is organized as follows. In the second section we define the problem and describe the optimization method as well as the IB technique. In the third section, a thorough discussion of the results of the optimization analysis is provided. Finally, concluding remarks are provided.

\section{Problem formulation}

\subsection{Governing equations and numerical method}

The dynamics of a three-dimensional incompressible flow is described by the following governing equations:

$$
\begin{gathered}
\mathbf{u}_{t}+(\mathbf{u} \cdot \nabla) \mathbf{u}=-\nabla p+\frac{1}{R e} \nabla^{2} \mathbf{u} \\
\nabla \cdot \mathbf{u}=0
\end{gathered}
$$


where $\mathbf{u}$ is the velocity vector and $p$ is the pressure term (including the contribution of conservative-force fields). Dimensionless variables are defined with respect to the Blasius displacement thickness measured at the abscissa corresponding to the center of the roughness element, $\delta^{*}$, and to the freestream velocity, $U_{\infty}$; therefore, the Reynolds number is $R e=U_{\infty} \delta^{*} / \nu, \nu$ being the kinematic viscosity. The reference computational domain has dimensions equal to $L_{x}=216, L_{y}=27.5$ and $L_{z}=72, x, y$ and $z$ being the streamwise, wall-normal and spanwise directions, respectively. The roughness element is an axisymmetric bump and its height is defined as $h(r)=k \cos ^{3}(\pi r / d)$, where $k$ is the maximum height, $r$ is the distance from the center of the bump on the $x-z$ plane, and $d$ is its diameter (see Piot et al. (2008)). Computations have been performed using five values of $k$, namely, $k=0.5,0.75,1,1.25,1.5$; whereas the diameter, $d=26$, has been kept constant. The roughness element is centered at $z=36$ in the spanwise direction, and it is placed 40.5 units downstream of the inlet section of the computational domain. For the three values of the Reynolds number considered here, namely $R e=170,235,300$, the inlet velocity corresponds to the Blasius profile at a distance of $x_{i n}=15,36.5,60$ units downstream of the leading edge of the plate, respectively.

The base flow is obtained by integrating the Navier-Stokes equations with the following boundary conditions: at inlet points, the Blasius boundary-layer profile is imposed for the streamwise and wall-normal components of the velocity vector whereas the spanwise component is set to zero. At outlet points, a standard convective condition is employed (Bottaro 1990). In the spanwise direction periodicity is imposed for the three velocity components. At the upper-boundary points, the Blasius solution is imposed for the wallnormal component of the velocity, whereas the spanwise velocity component and the spanwise vorticity are set to zero. Finally, at the bottom boundary, the no-slip boundary condition is prescribed using the IB technique. In particular, as shown in Figure 1, the body surface is overlapped onto the volume mesh, splitting it into fluid and solid regions, $\Gamma_{1}$ and $\Gamma_{2}$, respectively. The computational cells are assigned to each zone using a raytracing technique (O'Rourke 1993). At each point of the computational domain $\Gamma_{1}+\Gamma_{2}$, the governing equations (2.1) and (2.2) are solved, using a direct forcing technique at interface points to force the desired velocity value. A more detailed description of the IB technique is provided in Appendix A.

The governing equations are discretized by a finite-difference fractional-step method (Verzicco \& Orlandi 1996). The viscous terms are discretized in time using an implicit Crank-Nicolson scheme, whereas an explicit third-order-accurate Runge-Kutta scheme is employed for the non-linear terms. A second-order-accurate centered space discretization is used on a staggered grid. After a grid-convergence analysis, a mesh made up by $301 \times 150 \times 91$ points clustered towards the wall is selected for the computational domain.

\subsection{Lagrangian optimization}

The linear behavior of a perturbation $\mathbf{q}=\left(u^{\prime}, v^{\prime}, w^{\prime}, p^{\prime}\right)^{T}$ evolving in a laminar incompressible flow over a roughness element placed over a flat plate is studied by employing the governing equations linearized around the steady state $\mathbf{Q}=(U, V, W, P)^{T}$. A zero perturbation condition is chosen for the three velocity components at the $x$ and $y$ boundaries, whereas periodicity of the perturbation is imposed in the spanwise direction. The zero perturbation condition at the outflow is enforced by means of a fringe region, which allows the perturbation at the exit boundary to vanish smoothly.

In order to identify the perturbation at $t=0$ capable of producing the largest energy growth at any given target time, $T$, a Lagrange multiplier technique is used (see Zuccher 


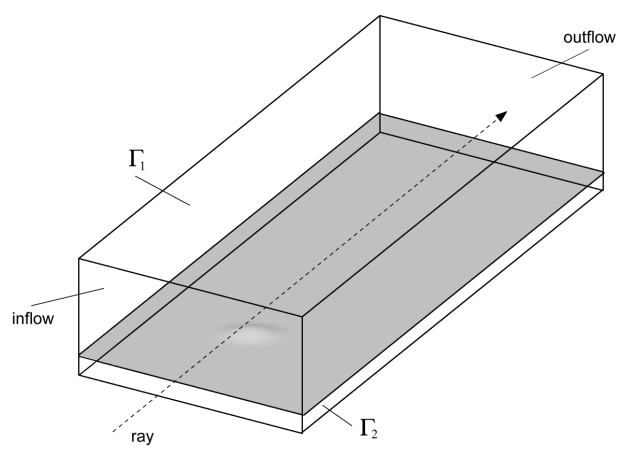

Figure 1. Sketch of the computational domain: $\Gamma_{1}$ and $\Gamma_{2}$ indicate the fluid and solid regions respectively, separated by the body surface, in gray.

et al. (2004)). Let us define the disturbance energy as:

$$
E(t)=\int_{V}\left(u^{\prime 2}(t)+v^{\prime 2}(t)+w^{\prime 2}(t)\right) d V
$$

where $V$ is the computational volume. The objective function of the procedure is the energy gain at the target time $t=T$, i.e. $E(T) / E(0)$. The Lagrange multiplier technique consists in seeking extrema of the augmented functional $\mathcal{L}$ with respect to every independent variable. Such a functional is written as:

$$
\begin{gathered}
\mathcal{L}=\frac{E(T)}{E(0)}+\int_{V} \int_{0}^{T} a\left(u_{x}^{\prime}+v_{y}^{\prime}+w_{z}^{\prime}\right) d t d V \\
+\int_{V} \int_{0}^{T} b\left(u_{t}^{\prime}+\left(u^{\prime} U\right)_{x}+U_{y} v^{\prime}+V u_{y}^{\prime}+U_{z} w^{\prime}+W u_{z}^{\prime}+p_{x}^{\prime}-\frac{u_{x x}^{\prime}+u_{y y}^{\prime}+u_{z z}^{\prime}}{R e}\right) d t d V \\
+\int_{V} \int_{0}^{T} c\left(v_{t}^{\prime}+U v_{x}^{\prime}+\left(v^{\prime} V\right)_{y}+u^{\prime} V_{x}+V_{z} w^{\prime}+W v_{z}^{\prime}+p_{y}^{\prime}-\frac{v_{x x}^{\prime}+v_{y y}^{\prime}+v_{z z}^{\prime}}{R e}\right) d t d V \\
+\int_{V} \int_{0}^{T} d\left(w_{t}^{\prime}+U w_{x}^{\prime}+V w_{y}^{\prime}+W_{x} u^{\prime}+W_{y} v^{\prime}+\left(W w^{\prime}\right)_{z}+p_{z}^{\prime}-\frac{w_{x x}^{\prime}+w_{y y}^{\prime}+w_{z z}^{\prime}}{R e}\right) d t d V
\end{gathered}
$$

where $a, b, c, d$ are the Lagrange multipliers (also called adjoint variables) and the linearized governing equations (also called direct problem) are the constraints, where the subscripts denote derivation. Integrating by parts and setting to zero the first variation of $\mathcal{L}$ with respect to $u^{\prime}, v^{\prime}, w^{\prime}, p^{\prime}$ leads to the adjoint equations and to the compatibility conditions, which are detailed in Appendix B. Enforcing the boundary conditions on the direct variables we obtain corresponding boundary conditions for the adjoint variables. For both the direct and adjoint equations, the zero perturbation condition at the wall is imposed by means of the IB technique (see Appendix A). Finally, the gradient of the augmented functional with respect to the initial perturbation is forced to vanish by means of a coupled iterative approach similar to that used by Marquet et al. (2008) and Cherubini et al. (2011), employing a conjugate gradient method. A detailed description of the optimization technique is provided in Appendix B. 


\begin{tabular}{llllll|l|l|l|l|}
\hline \multicolumn{1}{c|}{$\| k=0.50$} & $k=0.75$ & $k=1.00$ & $k=1.25$ & $k=1.50 \mid$ \\
\hline$R e=170 \|$ & 25.1 & $\mid$ & 57.5 & $\mid$ & 95.4 & 144.9 & 198.3 \\
\hline$R e=235 \|$ & 35.5 & $\mid$ & 77.6 & $\mid$ & 134.1 & 203.2 & 275.6 \\
\hline$R e=300 \|$ & 47.1 & $\mid$ & 99.7 & $\mid$ & 171.2 & $\mid$ & 252.3 & $\mid$ \\
\hline$R 51.5$
\end{tabular}

TABLE 1 . Values of $R e_{k}$ for all of the base flows considered.

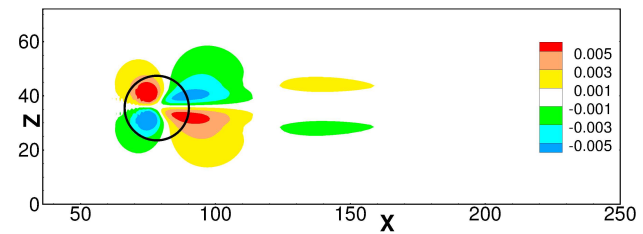

(a)

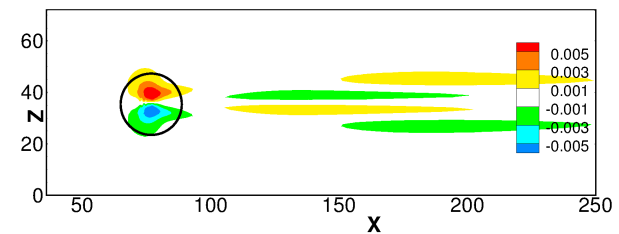

(b)

FIGURE 2. Contours of the streamwise vorticity in the plane $y=k+1$, for the two base flows with $k=0.75$ (a), and $k=1.5$ (b) at $R e=235$. The black circle represents the bump.

\section{Results}

\subsection{The base flow}

Steady base flows have been computed for three values of the Reynolds number, all of them being subcritical with respect to TS waves, using five bump heights, namely, $k=0.5,0.75,1,1.25,1.5$. Table 1 provides, for all of the considered base flows, the values of the roughness Reynolds number defined as $R e_{k}=U_{(y=k)} k / \nu\left(U_{(y=k)}\right.$ being the flow velocity at the point of maximum roughness height in absence of the roughness element). This is an important parameter related to the maximum streak amplitude (see White \& Ergin (2003)), providing a threshold for roughness-induced transition $\left(R e_{k} \approx 600\right.$, see Tani et al. (1962)). For the base flows considered here, the roughness Reynolds numbers range from a minimum value of 25.1 (for $R e=170$ and $k=0.5$ ) to a maximum value of 351.5 (for $R e=300$ and $k=1.5$ ), which is close to the $R e_{k}$ used by Fransson et al. (2005) in an experimental work on cylindrical roughness elements. As found in previous works (see Joslin \& Grosch (1995); Fransson et al. (2004); Piot et al. (2008)), in all cases we observe counter-rotating vortices downstream of the roughness element: Figure 2 provides the streamwise vorticity in the plane $y=k+1$ for the base flows at $R e=235$ with $k=0.75$ (a) and $k=1.5$ (b). As also pointed out by Fransson et al. (2005), it appears that the strength of the vortices is related to the roughness height with respect to the boundary-layer displacement thickness. Figure 2 (a) shows that the smaller bump is characterized by large vorticity regions in the near field downstream of the roughness element, mostly due to the wake of the bump, whereas, for the larger bump elongated streamwise vortices with a large development in the streamwise direction can be observed in Figure 2 (b). The streamwise vortices transport the high-speed fluid from the outer 

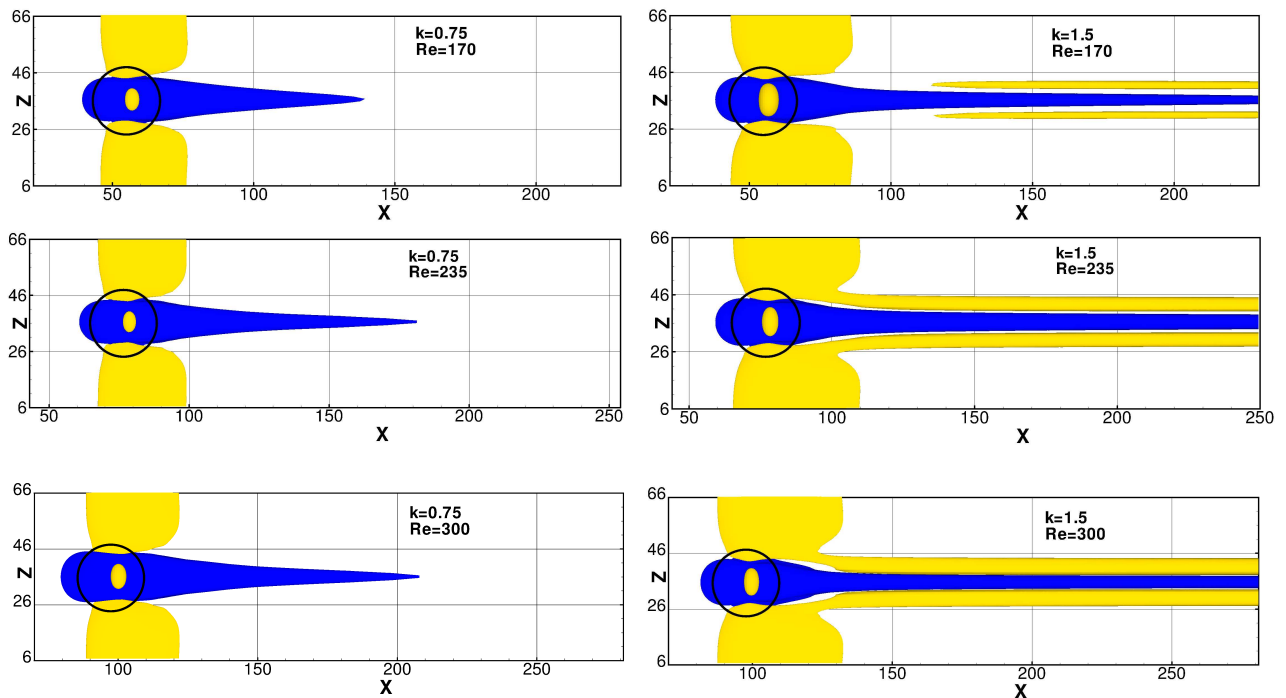

FIGURE 3. Surfaces of the positive (light grey, yellow online) and negative (dark grey, blue online) deviation of the streamwise component of velocity with respect to the spanwise-average value for two base flows at $R e=170$ (top frames), $R e=235$ (middle frames), and $R e=300$ (bottom frames) with $k=0.75$ (left frames, $\bar{u}= \pm 0.01$ ), and $k=1.5$ (right frame, $\bar{u}= \pm 0.018$ ). The black circle represents the bump.

region of the boundary layer towards the wall, whereas the low-speed fluid is transported away from the wall. This produces streamwise streaks which can be visualized by showing the difference between the streamwise component of the velocity and its mean value in the spanwise direction, $\bar{U}(x, y)=\frac{1}{L_{z}} \int U(x, y, z) d z$. The streamwise velocity deviation, $\bar{u}(x, y, z)=u(x, y, z)-\bar{U}(x, y)$, is provided in Figure 3 for $R e=170,235,300$ (top, middle and bottom frames, respectively), with $k=0.75$ (left frames) and $k=1.5$ (right frames). In the latter case, the streamwise streaks developing into the boundary layer are clearly seen. For a given $k$, the base flows obtained for different Reynolds numbers show rather similar structures and velocity amplitudes. Thus, the base flow appears to depend more on the height of the bump than on its position in the streamwise direction. For small bump's heights, low- and high- momentum regions are localized close to the roughness element; whereas, for large bump heights, one can observe that three confining streaks are generated downstream of the bump. The streamwise velocity deviation has a large negative amplitude in the vicinity of the bump, reaching values up to $\bar{u}(x, y) \approx-0.2$ at $x \approx 150$ for $R e=300$, but it tends to weaken as it develops in the streamwise direction $(\bar{u}(x, y) \approx-0.13$ at $x \approx 250)$. Being strongly dependent on the streamwise abscissa, these low-momentum structures are rather different from the quasi-parallel streaks representing the local optimal perturbations in a boundary layer flow (Luchini 2000). The presence of a negative streamwise streak following the wake of the bump is consistent with previous experimental data on the flow past a shallow bump (see Joslin \& Grosch (1995); Gaster et al. (1994)). On the other hand, many experimental results for cylindrical roughness elements (see Fransson et al. $(2004,2005)$ ) show a positive streak following the wake, due to the coalescence of the two positive streaks generated by the horseshoe vortex. This difference is probably due to the large diameter of the bump considered here (in terms of the boundary-layer displacement thickness) which hampers the coalescence of the two positive streaks. It is noteworthy that the different structure of the base flow precludes the generalization of the results of the stability analysis obtained here to roughness elements 
of any shape and aspect ratio.

The negative streak is flanked by two positive streaks which are characterized by lower amplitudes and present a weak dependence on the streamwise direction, reaching at $x \approx 170$ values of the deviation from the mean up to $\bar{u}(x, y) \approx 0.07$ for $R e=235,300$, and $\bar{u}(x, y) \approx 0.02$ for $R e=170$. This is consistent with previous experimental results (White \& Ergin 2003; Fransson et al. 2004) showing that, downstream of the roughness element, the three-dimensional flow evolves gradually into a quasi-parallel streaky structure. The distance from the bump at which the streaks become quasi-parallel may depend on the configuration parameters. However, in our computations, as well as in the results by Piot et al. (2008) and Joslin \& Grosch (1995), quasi-parallel streaks are observed at about 100 length units downstream of the roughness location. It is noteworthy that, even if in the vicinity of the bump the flow presents strong modifications with respect to the undisturbed boundary-layer flow, in the downstream zone, where the effect of the wake is weakened, the amplitudes of the streaks do not exceed the threshold value for secondary instability $\left(u^{\prime} \approx 0.23\right.$, see Andersson et al. (2001)).

\subsection{Optimal perturbations}

\subsubsection{The optimal energy gain}

Direct-adjoint optimizations have been performed for all of the base flows described above. Figures 4 (a), (b), and (c) provide the value of the optimal energy gain versus the target time for the Blasius base flow (dotted lines), and for the base flows in the presence of the bumps of different heights obtained at $R e=170, R e=235$, and $R e=300$, respectively, in the reference computational domain. One can observe that, in the presence of the roughness element, the energy gain is always larger than the one obtained for the Blasius base flow, although for small roughness heights $(k<1)$ the differences are negligible. For $k \geqslant 1$, a non negligible increment in the energy gain is observed, which grows substantially with the bump height, overtaking the energy of the Blasius case up to two orders of magnitude, for $k=1.5$ and $T=100$. It is noteworthy that in all cases the largest differences are observed at small times, indicating that a strong transient growth mechanism exists, acting very fast in time. Since Figures 4 (a), (b), and (c) show that the energy gain always increases with $k$, one can infer that the larger the streak amplitude in the base flow, the stronger the transient growth of the energy. However, a strong increase of the energy gain is also observed increasing the Reynolds number at given $k$. Since in these cases, very similar shape and values of the steady streaks are observed (see Figure 3 ), we can infer that not only the streak amplitude, but also the streamwise position of the roughness element is an important parameter of the transient growth mechanism.

\subsubsection{The effect of the bump height}

The differences in the energy gain values correspond to different shapes and localizations of the initial optimal perturbations. Figures 5 (a)-(d) show the iso-surfaces of the spanwise velocity component of the initial optimal perturbation for the Blasius flow $(k=0)$ and for $k=0.5,1.0,1.5$, respectively, at a small target time, $T=50$, and $R e=235$. For the Blasius flow, as already found by the global optimizations by Cherubini et al. (2010b) and Monokrousos et al. (2010), the initial optimal perturbation is composed of upstream-inclined vortices, aligned with the $x$-axis, alternated in the streamwise and spanwise direction. Since the Blasius flow is homogeneous in the spanwise direction, the result of the optimization contains only one spanwise wavenumber, $\beta$. At $T=50$, the spanwise wavenumber of the optimal perturbation is $\beta=0.87$ and is found to decrease for larger target times, reaching at the optimal target time (i.e., the time at which the 


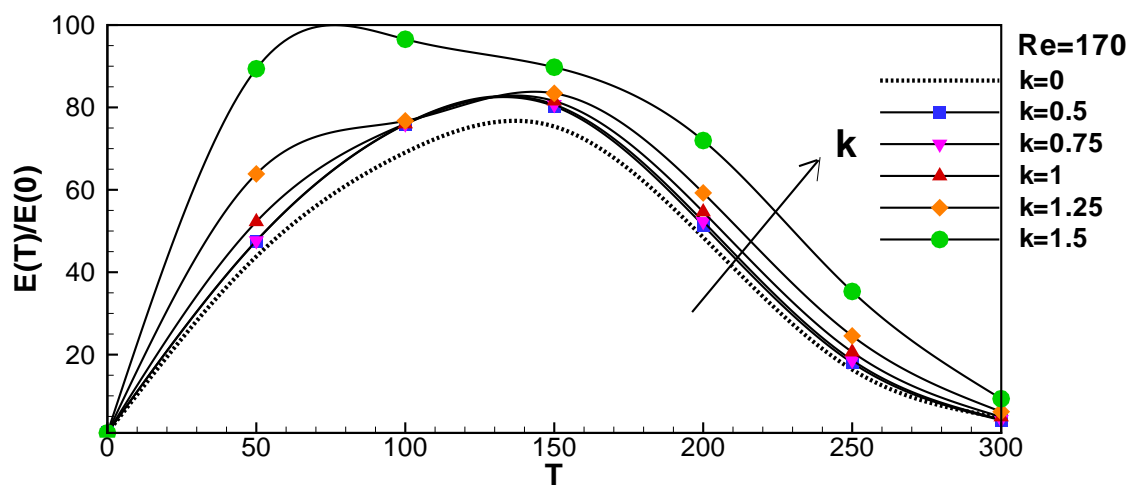

(a)

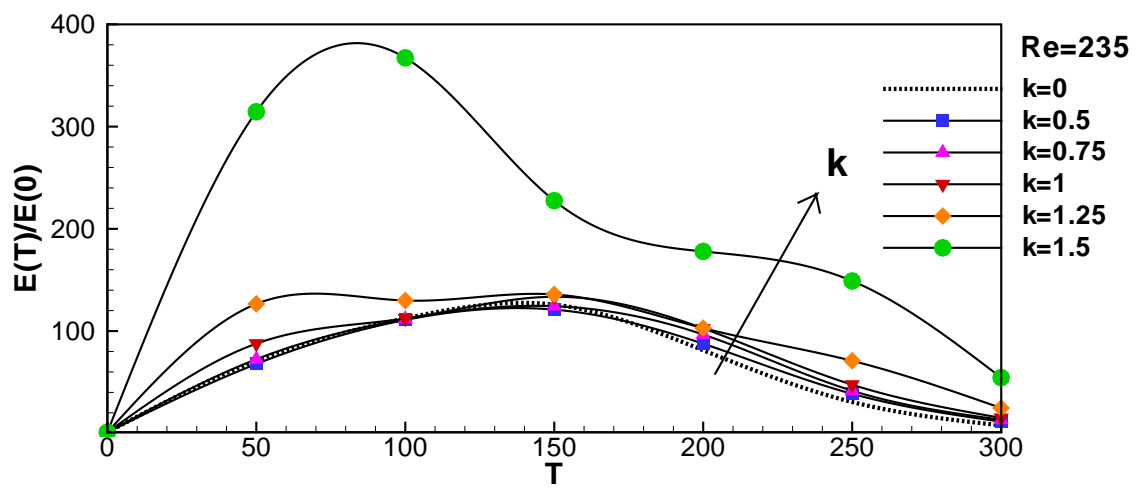

(b)

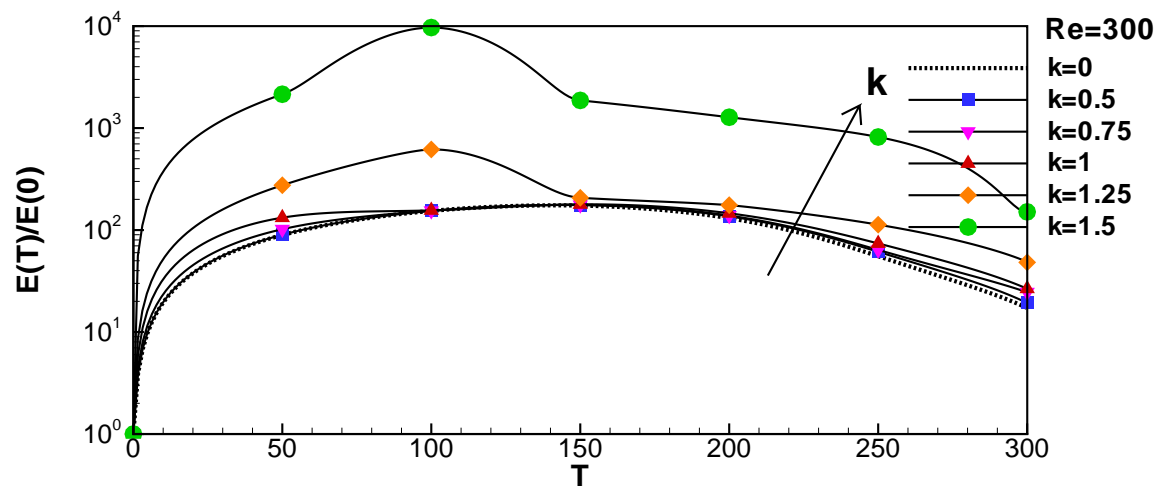

(c)

FiguRE 4. Optimal energy gain versus target time for the Blasius base flow (dotted line) and in the presence of the bumps of different heights (increasing bump height following the arrow), for $R e=170$ (a), $R e=235$ (b), and $R e=300$ (c). The symbols correspond to: $k=0.5$ (squares), $k=0.75$ (gradients), $k=1$ (deltas), $k=1.25$ (diamonds), and $k=1.5$ (circles). 
maximum amplification is reached) a value which is close to the optimal wave number computed locally by Corbett \& Bottaro (2000), namely, $\beta=0.6$. In the presence of the roughness element, the base flow is not homogeneous in the spanwise direction, therefore the optimal perturbation does not show only one wavenumber $\beta$. Indeed, for all of the bump heights, the optimal disturbance is localized in the spanwise direction, being composed of pairs of upstream-inclined vortices, aligned with the $x$-axis, alternated in the spanwise and streamwise direction, and characterized by a dominant spanwise wavelength which is slightly larger than that of the Blasius flow. For increasing bump height, the spanwise wavelength of the initial vortices increases, and the perturbation appears to be more flat and spatially localized, moving upstream towards the top of the roughness element. This indicates that the fast transient growth observed for large bump heights at small target times is mostly linked to the wake of the bump, rather than to the streaks generated downstream. The structure of the optimal perturbation for small bump height is very similar to the optimal one recovered for the Blasius flow (see Cherubini et al. $(2010 b))$, although the perturbation is spanwise-localized. It is also noteworthy that, as in the case of the boundary layer (see Luchini (2000); Cherubini et al. (2010b)), at the initial time, the streamwise component of the perturbation is the smallest one, and the spanwise component is the largest. The amplitudes and the shape of the perturbation change for the case of the larger bumps, since the initial perturbation is characterized by large values of the streamwise component of velocity, and slightly smaller values of the spanwise and wall-normal ones. Moreover, the vortices have a larger inclination with respect to the streamwise direction. It is noteworthy that such an optimal disturbance presents some similarities with respect to the non-linear optimal perturbation found for the boundary-layer flow concerning the inclination of the vortices and the large initial amplitude of the streamwise perturbation, see Cherubini et al. $(2010 a, 2011)$. This indicates that, in the presence of a three-dimensional flow induced by a large roughness element, the amplification mechanisms producing a transient growth in a boundary-layer remarkably change.

An insight in such amplification mechanisms can be achieved by analyzing the shape of the optimal perturbations at the target time of the optimization $(T=50)$. Figures 6 (a)-(d) show the streamwise velocity component of the optimal perturbation at the target time for $k=0,0.5,1,1.5$, respectively, with $R e=235$. For the Blasius flow (Figure 6 (a)), the optimal perturbation is composed of streaky structures alternated in the streamwise and spanwise directions, preserving the same wavelength found at $t=0$ (compare with Figure 5), although such structures change their streamwise inclination. In fact, the perturbation tilts downstream via the Orr mechanism (Orr 1907) and is amplified by means of the lift-up effect (Butler \& Farrell 1992; Schmid \& Henningson 2001).

The optimal perturbation for the case of small bumps (see Figure 6 (b)-(c)), is similar to that obtained for a Blasius flow, although three main differences can be noticed: i) the perturbation moves upstream with respect to the Blasius case; ii) the perturbation localizes in the spanwise direction in a narrow zone close to the bump location; iii) the vortices are not aligned with the $x$-axis, showing a slight inclination with respect to the streamwise direction, and form an arrow-shaped wave packet.

For the case of the larger bump $(k=1.5$, see Figure $6(\mathrm{~d}))$, the structure of the perturbation at target time is characterized by the presence of "arches" at the center of the wave-packet. This perturbation has a stronger spanwise and streamwise localization with respect to the smaller bump case, with a larger inclination with respect to the streamwise direction. Moreover, one can clearly observe a varicose symmetry (see Schmid \& Henningson (2001)) with respect to the central negative streak.

The shape of such optimal perturbations is quasi-invariant with respect to the domain 


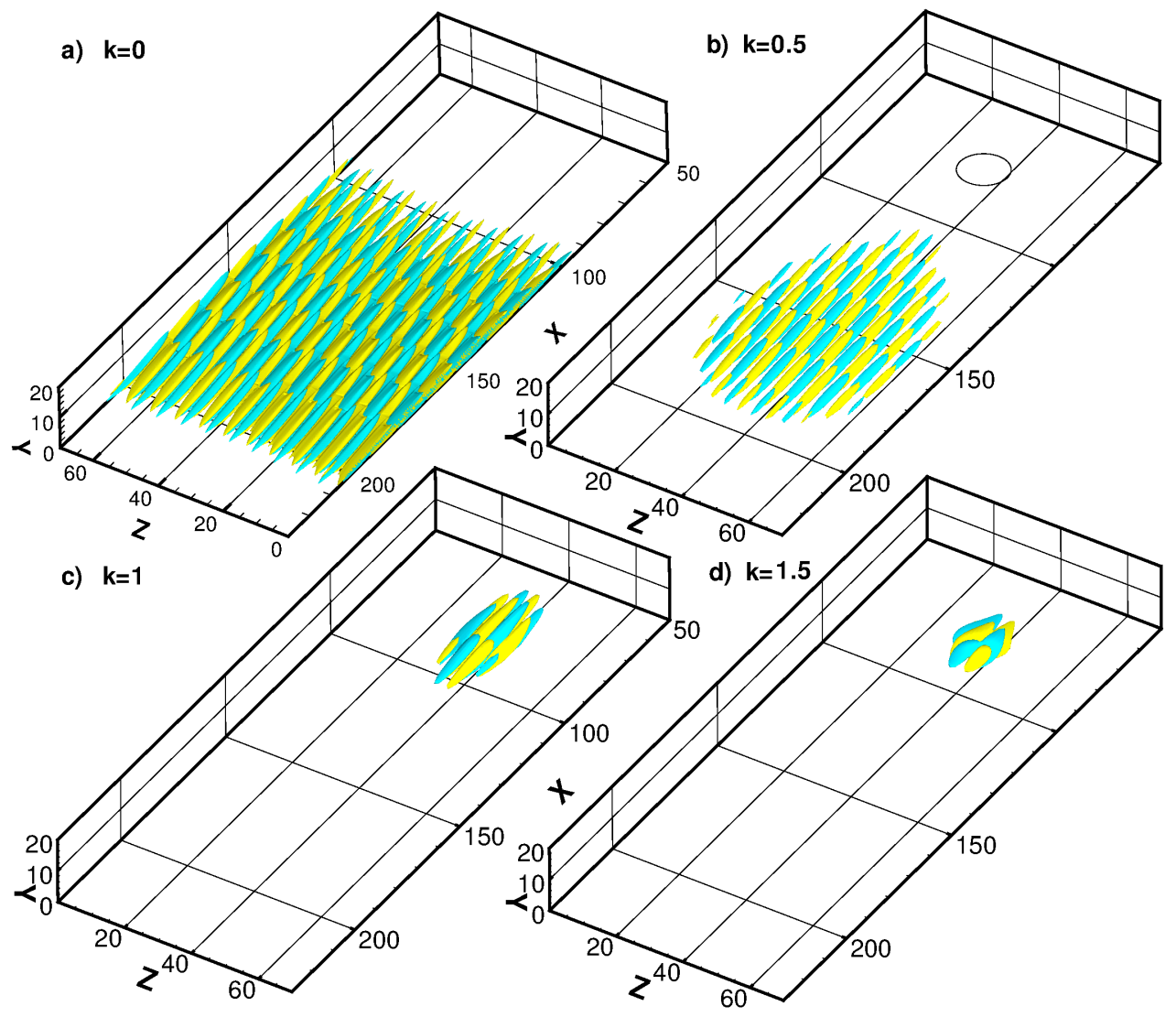

FIGURE 5. Iso-surfaces of the spanwise component of velocity (dark grey, blue online, and light grey, yellow online, for negative and positive values, respectively) for the initial optimal perturbations obtained at $T=50$ with $R e=235$ and $k=0$ (a), $k=0.5$ (b), $k=1$ (c), and $k=1.5$ (d).

lengths (for long enough domains and short enough target times), whereas the associate value of the energy gain may change remarkably. Figure 7 (a) shows that, for a too short streamwise length $\left(L_{x}=108\right.$, half of the reference one), the energy gain is lower with respect to the reference domain $\left(L_{x}=216\right)$ and suddenly drops, whereas it increases for a larger domain $\left(L_{x}=324\right)$. This is due to the convective nature of the transient growth mechanism. For larger Reynolds numbers and bump heights, changing the domain induces smaller differences in the amplification values at short target times, as shown in Figure 7 (b) for $R e=300$ and two different bump heights, $k=0.75$ and $k=1.5$. This is clearly due to the stronger localization of the optimal perturbation close to the bump. Finally, as provided in Figure 7 (c), the energy gain values is slightly dependent on the spanwise domain length, due to the strong localization of the optimal perturbation in the spanwise direction.

\subsubsection{The effect of the target time}

In the previous subsection, we have shown that optimal perturbations are strongly dependent on the bump height. In particular, we have identified two different types of optimal disturbances: i) the wave packet of quasi-streamwise vortices, not localized on the bump, shown in Figure 5 (b) for $R e=235, k=0.5$ and $T=50$, which for 


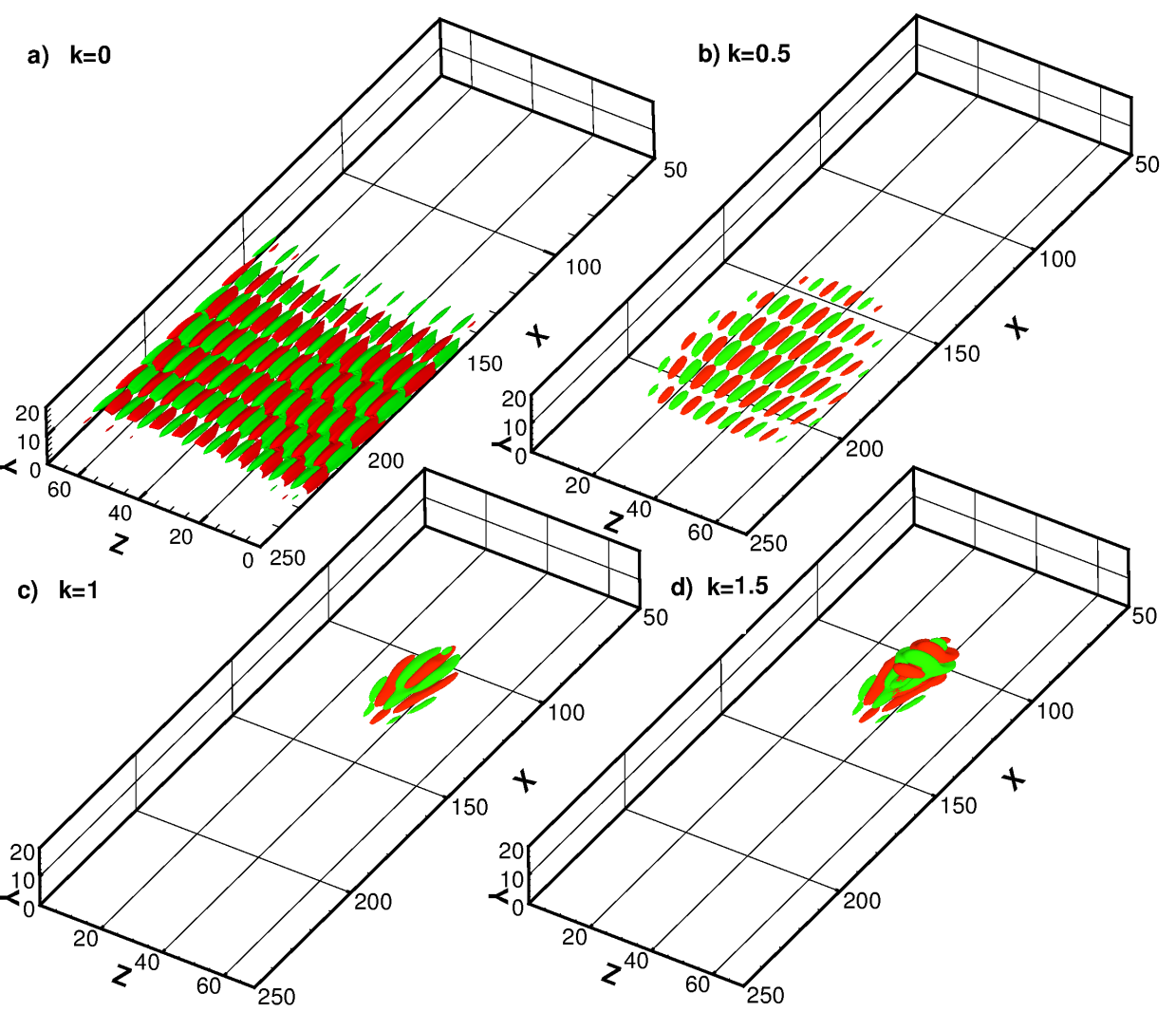

FiguRE 6. Iso-surfaces of the streamwise component of velocity (light grey, green online, and dark grey, red online, for negative and positive values, respectively) for the optimal perturbations at target time $T=50$ with $R e=235, k=0$ (a), $k=0.5$ (b), $k=1$ (c), and $k=1.5$ (d).

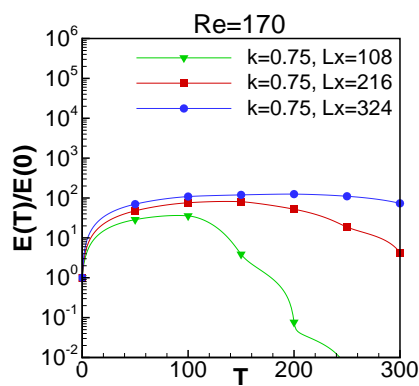

(a)

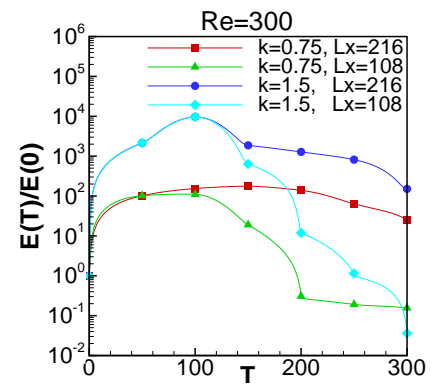

(b)

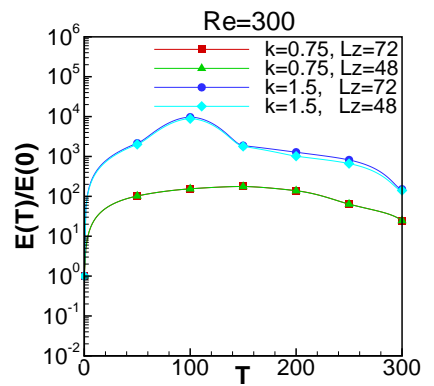

(c)

FiguRE 7. Optimal energy gain versus target time obtained with: (a) $R e=170$ and $k=0.75$, for three values of the streamwise domain length, namely, $L_{x}=108, L_{x}=216$ (the reference one), and $L_{x}=324$; (b) $R e=300, k=0.75$ and $k=1.5$, for two values of the streamwise domain length, namely, $L_{x}=108,216$; (c) $R e=300, k=0.75$ and $k=1.5$, for two values of the spanwise domain length, namely, $L_{z}=48, L_{z}=72$ (the reference one).

brevity will be thereafter referred to as "wave-packet perturbations" (WP); ii) the optimal perturbations having a more localized shape, above the roughness element, and presenting a varicose structure, such as the one in Figure 5 (d), which will be referred to as "varicose perturbations" (VP). In this subsection, we will investigate how the target time of the 


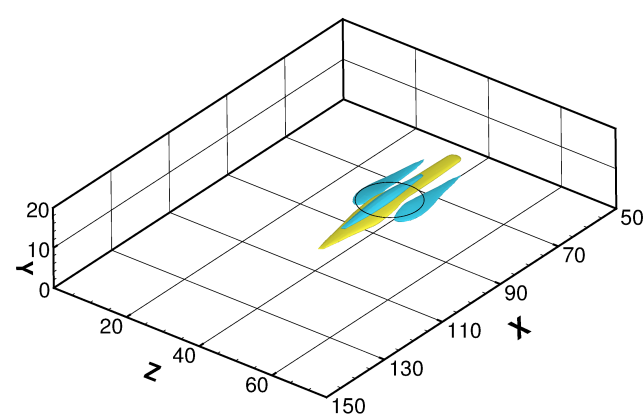

(a)

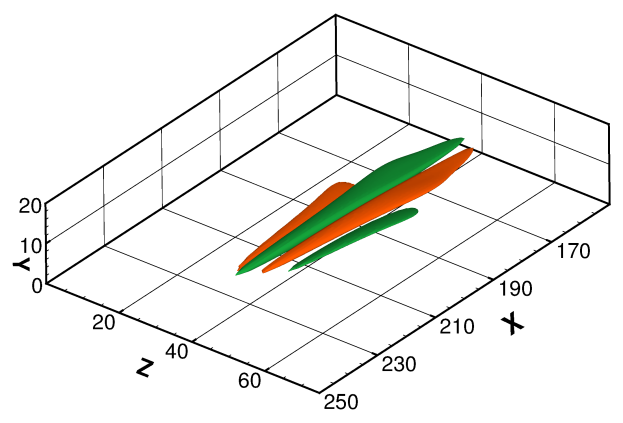

(b)

FiguRE 8. Iso-surfaces of the spanwise component of velocity (dark grey, blue online, and light grey, yellow online, for negative and positive values, respectively), and of the streamwise component of velocity (light grey, green online, and dark grey, red online, for negative and positive values, respectively) for the optimal perturbation at $t=0$ (a) and $t=T$ (b) obtained at $R e=235, T=200$ and $k=1.5$.

optimization affects the shape and structure of such optimal perturbations.

For small bumps $(k<1)$, when the target time is increased, the optimal WP maintains its shape and structure. In particular, the wavepacket of quasi-streamwise vortices moves upstream, as a consequence of the larger amount of time available for amplifying the energy, reaching the bump location for $T \geqslant 250$. Moreover, its main wavelength increases (as also observed in the Blasius case), leading also to an increase of the spanwise extention of the wave packet. On the other hand, for larger bumps, an increase of the target time is shown to induce remarkable differences in the structure of the optimal perturbation. Figure 8 (a) provides the iso-surfaces of the spanwise velocity component of the initial optimal perturbations obtained for $R e=235$ with $T=200$ and $k=1.5$. We can observe that at the initial time the vortices are very elongated and alternated in the wall-normal direction. The spanwise component of the velocity perturbation appears to be symmetric with respect to the $x-y$ plane cutting the bump at its center, whereas at $T=50$ it was antisymmetric with respect to this plane (see Figure 5). Moreover, the largest component of the perturbation velocity at initial time is not the streamwise one, as in the VP case, but the spanwise one. At target time, the optimal perturbation (Figure 8 (b)) is an arrow-shaped wave-packet composed of spanwise-alternated streaky structures being characterized by a finite inclination with respect to the streamwise direction. The "arch" structures observed at smaller target time (see Figure 6 (d)) are not found in the present case, and the streamwise component of the velocity perturbation is antisymmetric with respect to the $x-y$ plane cutting the bump at its center (whereas it was symmetric with respect to such a plane for a smaller target time). It appears that the optimal disturbance obtained for $T=200$ in the case of the large bump at $R e=235$ is a perturbation characterized by sinuous symmetry (see Schmid \& Henningson (2001)), whereas for $T=50$ it was a varicose perturbation. The different shape of the perturbation is linked to the drop of the energy gain observed in Figure 4 (b) for values of the target time between 100 and 150. The differences between the optimal disturbances for $R e=235$ and $k=1.5$ at "small" $(T \leqslant 100)$ and "large" $(T>100)$ target time can be better observed in Figures 9 (a)-(b), which provide the streamwise velocity component and the cross-stream vectors for the initial optimal disturbances obtained at $T=100$ and $T=150$, respectively, on the plane $x=77.5$. Despite the fact that the two target times are close to each other, the optimal disturbances change strongly in shape, showing: 


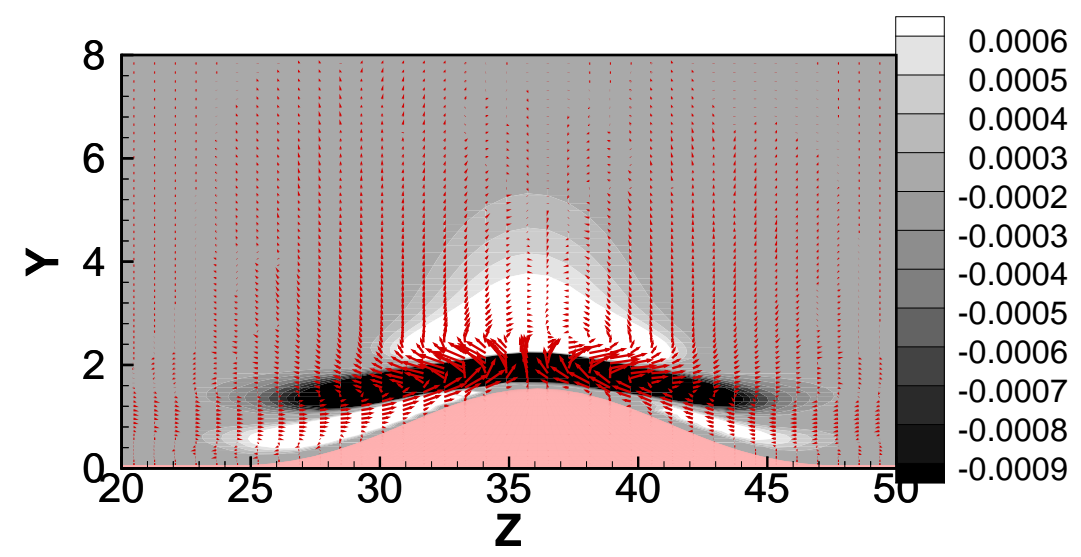

(a)

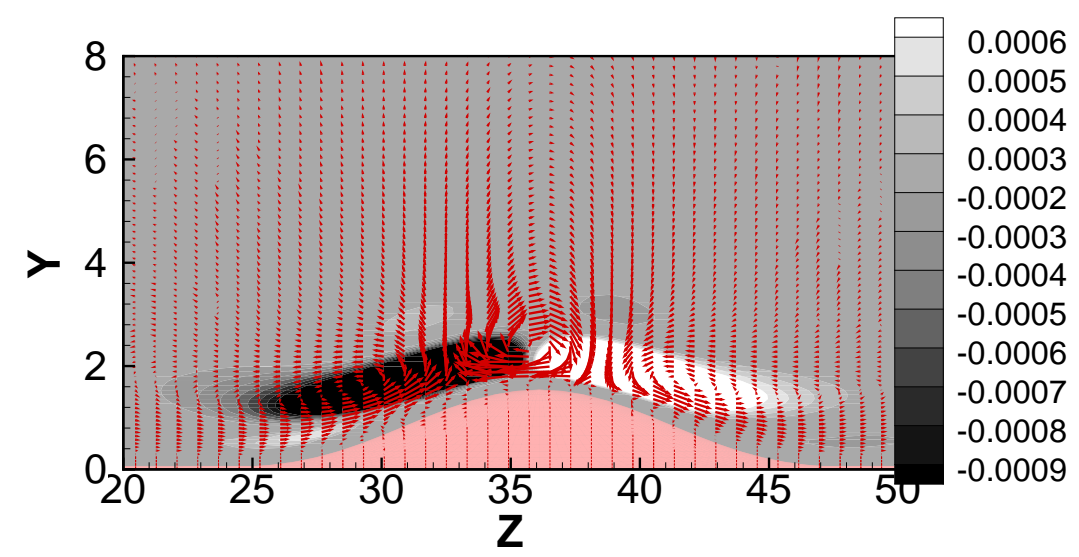

(b)

Figure 9. Cross-stream vectors and streamwise velocity component contours in the plane at $x=77.5$ for the initial optimal perturbation obtained at $T=100$ (a) and $T=150$ (b) for $R e=235$ and $k=1.5$. The spanwise and wall-normal axis are not on the same scale.

i) an "arch" structure for $T=100$, with wall-normal alternated patches of streamwise disturbance characterized by a varicose symmetry, and two vortices on the sides of the bump; ii) a flat structure for $T=150$, with spanwise-alternated patches of streamwise disturbance characterized by a sinuous symmetry, a main vortex on the top of the bump and two weaker vortices on its sides. Similar differences are recovered at target time, as shown in Figure 10 (a)-(b) on two $x$-constant planes $(x=135$ for $T=100$ and $x=165$ for $T=150$ ). However, the two perturbations also show some similarities, since they both have their maximum values in the zones of maximum shear of the base flow, represented in the figure by the solid contours of the streamwise component of velocity. Moreover, in both cases, the streamwise component of velocity is the most amplified, and its amplitudes are found to be similar both at $t=0$ and at $t=T$, although the optimal energy gain for $T=150$ is smaller than the one obtained for $T=100$. Such features closely recall the varicose and sinuous (respectively) local optimal perturbations found in Hoepffner et al. (2005) for a base flow composed of parallel saturated streaks. However, in such a 
parallel case, sinuous perturbations always provided the largest energy growth, whereas in the three-dimensional case considered here, varicose perturbations are found to be optimal for smaller target times, and sinuous perturbations for larger target times. The reason of this symmetry-change can be explained by observing that the base flow itself is characterized by a varicose symmetry in the near field downstream of the bump, whereas, further downstream, it turns into a nearly-parallel streaky flow (see the right frames of Figure 3). Thus, at small target times the optimal disturbances are strongly affected by the varicose structure of the base flow, inducing a varicose non-normal instability, whereas they turn into sinuous ones when they reach the nearly parallel streaky flow far from the bump. The fact that, for a high enough bump, the largest energy growth is obtained for a varicose perturbation, is an important result with respect to the wellknown result of the secondary instability of boundary layer streaks, assessing that the primary instability of a streaky flow is of sinuous type (Andersson et al. 2001). In fact, we have shown that in a realistic case in which streaks are generated by a smooth roughness element, the three-dimensionality of the flow is able to trigger a strong mechanism of transient amplification which can by-pass the asymptotical secondary instability of the streaks.

\subsubsection{The effect of the Reynolds number}

We have shown that, for different Reynolds numbers and the same bump height, the base flows are rather similar to each other (compare the top, middle and bottom frames of Figure 3). However, the remarkable increase of the value of the energy gain with the Reynolds number (especially in the case of large bump heights) indicates that large differences in the optimal perturbations should exist. Thus, the optimal perturbations for all of the considered Reynolds numbers, target times, and bump heights have been analyzed. All of the perturbations have been found to show a shape similar to that analyzed in the previous subsection, so that they can be roughly categorized as "wave packet perturbation" (WP), "varicose perturbation" (VP), and "sinuous perturbation" (SP). The complete result map is shown in table 2, where the subscript indicates the values of $R e$ (where no subscript is given, the result is valid for all $R e$ ), and bold indicates the optimal target time. As one can observe, for bump heights lower than $k=1$, for all values of the Reynolds numbers, the optimal perturbations are of the "wave packet" type, inducing an energy gain only slightly larger than that found for the Blasius flow (see Figures 4 (a)(b)-(c)). On the other hand, for larger bump heights the situation changes, since sinuous and varicous perturbations are found for most values of the target time and Reynolds number. In particular, for the lowest Reynolds number considered here, i.e., $R e=170$, the VP is never observed, and the WP is observed up to larger bump heights $(k=1.25)$. Moreover, for $k=1.5$ the optimal perturbation is a SP indipendently of the target time. Thus, it appears that for a too low Reynolds number, the transient instability of the wake linked to varicose optimal perturbations cannot be triggered, probably because the three-dimensionality of the flow is too weak. On the other hand, for $R e=235$, 300, the shape and structure of the optimal perturbations for each value of $T$ and $k$ are the same, characterized, for $k>1$, by a varicous/sinuous symmetry at small/large target times. This could be anticipated since the base flows at these two Reynolds numbers are very close to each other, although they are characterized by different values of $R_{k}$ (see table 1). This indicates that the structure of the optimal perturbation does not depend on $R e_{k}$, but rather on the particular three-dimensional shape of the flow. On the other hand, the energy gain increases dramatically with the Reynolds number for target times and bump heights related to varicose perturbations, even when the base flows are very similar to each other (for instance, compare the middle and bottom frames in Figure 


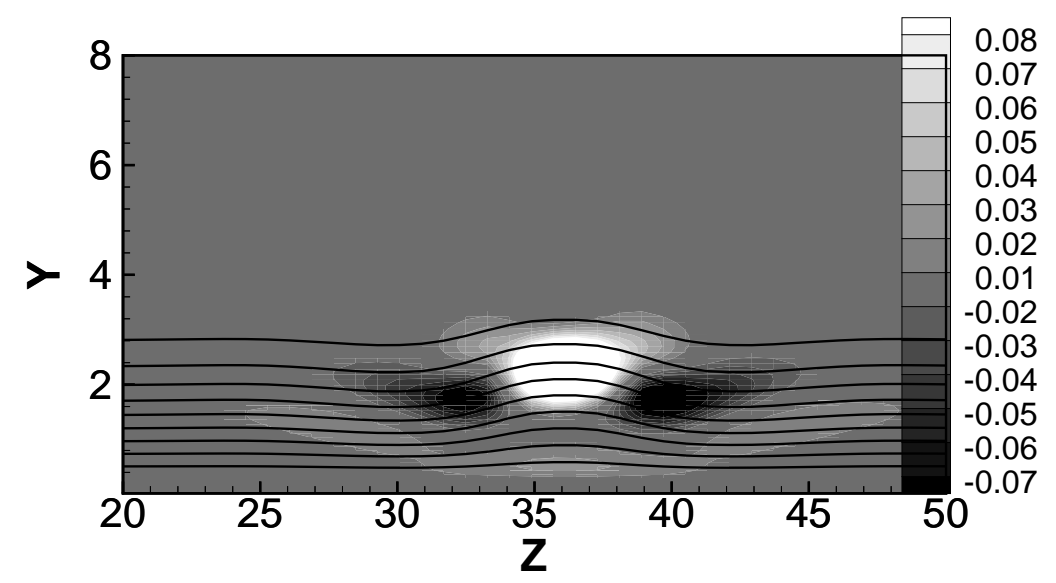

(a)

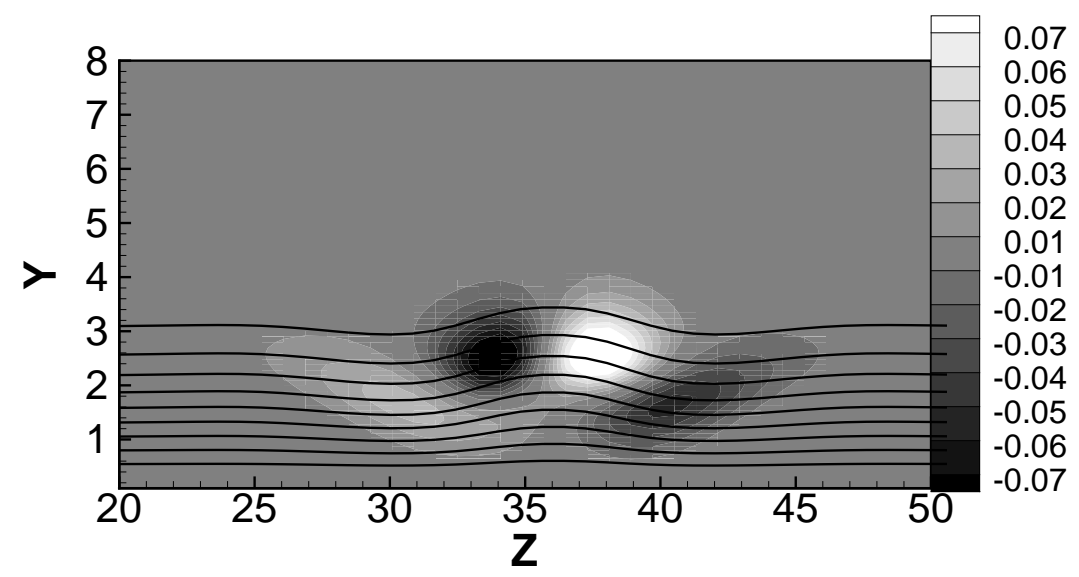

(b)

FiguRE 10. Shaded contours of the streamwise velocity component of the optimal perturbation at target time $T=100$ (a) and $T=150$ (b) in the planes $x=135$ (a) and $x=165$ (b) with $R e=235$ and $k=1.5$. The solid contours represent the streamwise component of the base flow velocity in the range $[0.1,0.9]$. Axis are not on the same scale.

4). This means that, even if the shape of the initial optimal disturbance does not depend on $R e_{k}$, the energy gain does. Thus, the transient instability induced by this type of perturbations becomes much stronger when the Reynolds number and the roughness height is increased, meaning that for large enough bumps such effect might overtake the stabilizing effect operated on the Tollmienn-Schlichting waves.

\subsubsection{The linear amplification mechanisms}

In this section we will analyze in detail the amplification mechanisms related to the sinuous and varicose optimal perturbations. To this end, we choose two optimal perturbations, obtained for the same Reynolds number and roughness height $(R e=300$, $k=1.5$ ), for two different target times: $T=50$ for the VP, and $T=200$ for the SP (see table 2). For brevity, in what follows we will refer to these two perturbations simply as $\mathrm{VP}^{*}$ and $\mathrm{SP}^{*}$.

Figure 11 shows the evolution of $\mathrm{VP}^{*}$, extracted at different times from a linearized 
Transient growth in the flow past a three-dimensional smooth roughness element 17

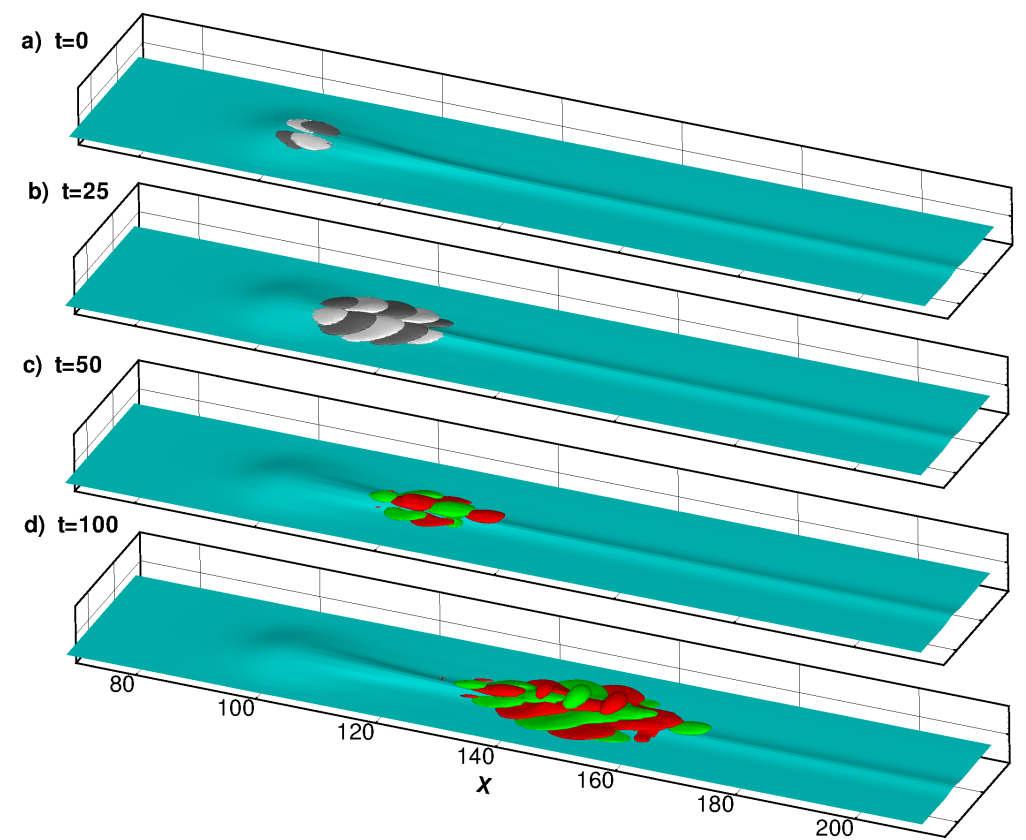

FIGURE 11. Evolution in time of the linear optimal perturbation obtained for $R e=300, k=1.5$, $T=50$, with initial energy $E_{0}=10^{-9}$ : streamwise component of the base flow velocity (flat light blue surface for $U=0.2$ ); streamwise vorticity perturbation (white and black surfaces for $\omega_{x}= \pm 0.001$ ) at $t=0$ (a) and $t=25(\mathrm{~b})$, streamwise velocity perturbation (light grey, green online, and dark grey, red online, for $\left.u^{\text {prime }}= \pm 0.00002\right)$ at $t=50$ (c) and $t=100$ (d).

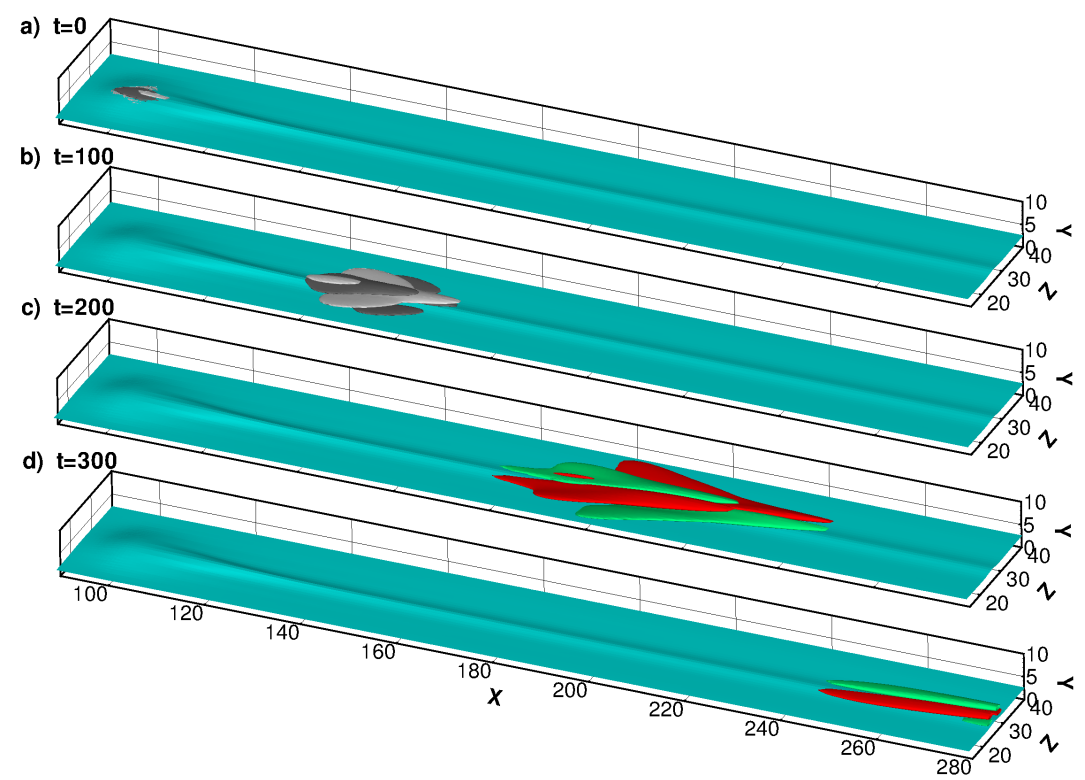

FIGURE 12. Evolution in time of the linear optimal perturbation obtained for $R e=300, k=1.5$, $T=200$, with initial energy $E_{0}=10^{-9}$ : streamwise component of the base flow velocity (flat light blue surface for $U=0.2$ ); streamwise vorticity perturbation (white and black surfaces for $\omega_{x}= \pm 0.00001$ ) at $t=0$ (a) and $t=100$ (b), streamwise velocity perturbation (light grey, green online, and dark grey, red online, for $\left.u^{\prime}= \pm 0.00001\right)$ at $t=200$ (c) and $t=300$ (d). 


\begin{tabular}{|c|c|c|c|c|c|c|}
\hline & $T=50$ & $T=100$ & $T=150$ & $=20$ & \urcorner$=2$ & $=3$ \\
\hline$k=0.50$ & WP & WP & WP & WP & WP & WP \\
\hline$k=0.75$ & WP & WP & WP & WP & WP & WP \\
\hline$k=1.00$ & $\mid \begin{array}{c}\mathrm{SP}_{170} \\
\mathrm{VP}_{235,300}\end{array}$ & WP & WP & WP & WP & WP \\
\hline$k=1.25$ & $\mid \begin{array}{c}\mathrm{SP}_{170} \\
\mathrm{VP}_{235,300}\end{array}$ & $\begin{array}{c}\mathrm{WP}_{170} \\
\mathbf{V P}_{\mathbf{2 3 5}, \mathbf{3 0 0}}\end{array}$ & $\mid \begin{array}{c}\mathbf{W P} \mathbf{P}_{\mathbf{1 7 0}} \\
\mathrm{VP}_{235,300}\end{array}$ & WP & $\mathrm{SP}$ & $\mathrm{SP}$ \\
\hline$k=1.50$ & $\mid \begin{array}{c}\mathrm{SP}_{170} \\
\mathrm{VP}_{235,300}\end{array}$ & $\begin{array}{c}\mathbf{S P}_{170} \\
\mathbf{V P}_{235,300}\end{array}$ & $\mid \begin{array}{c}\mathrm{SP}_{170} \\
\mathrm{VP}_{235,300}\end{array}$ & $\mathrm{SP}$ & $\mathrm{SP}$ & $\mathrm{SP}$ \\
\hline
\end{tabular}

TABLE 2. Optimal perturbations obtained for all of the base flows (WP meaning "wave packet perturbation", SP meaning "sinuous perturbation"). The subscripts indicate the Reynolds number; where no subscript is given the results are the same for all Re. Bold fonts indicate the optimal target time.

DNS. The initial antisymmetrically-alternated vortices (black and white surfaces) are tilted in the streamwise direction. At $t=25$ (Fig. 11 (b)), the vortices increase their amplitude and their extension in the spanwise direction, starting to generate streamwise perturbations by transporting the base flow shear (Fig. 11 (c) for $t=50$ ). At $t=100$, the streamwise perturbation has increased its amplitude and extention, assuming an arrow shape with the formation of little arches connecting the symmetric patches of perturbation. A similar picture is obtained for $\mathrm{SP}^{*}$, with important differences in the symmetry of the structures. Figure 12 (a) shows that the initial vortices are symmetric with respect to the streamwise direction and alternated in the wall-normal direction. They are tilted in the streamwise direction and inclined in the spanwise one, assuming an arrow shape at $t=100$ (Fig. 12 (b)). The streamwise perturbation generated by transport of the base flow has a similar shape, but the alternated streaky structures are antisymmetric with respect to the streamwise direction (Fig. 12 (c) and (d)). We can observe that, in both cases, tilting downstream the disturbance extracts energy from the mean shear due to the conservation of the circulation. This non-modal amplification mechanism is called Orr mechanism (Orr (1907); Butler \& Farrell (1992)), and is the only one present in a linear framework in the case of spanwise-independent perturbations in a shear flow. However, since the maximum growth is not attained when the disturbance is aligned in the wall-normal direction (Butler \& Farrell 1992), other mechanisms should be active to induce the strong amplification observed here. In order to identify the mechanisms inducing such a growth, we can use the Reynolds-Orr equations (Schmid \& Henningson 2001):

$$
\frac{d E}{d t}=-\int_{V} u_{i} u_{j} \frac{\partial U_{i}}{\partial x_{j}} d V-\frac{1}{R e} \int_{V} \frac{\partial u_{i}}{\partial x_{j}} \frac{\partial u_{i}}{\partial x_{j}} d V
$$




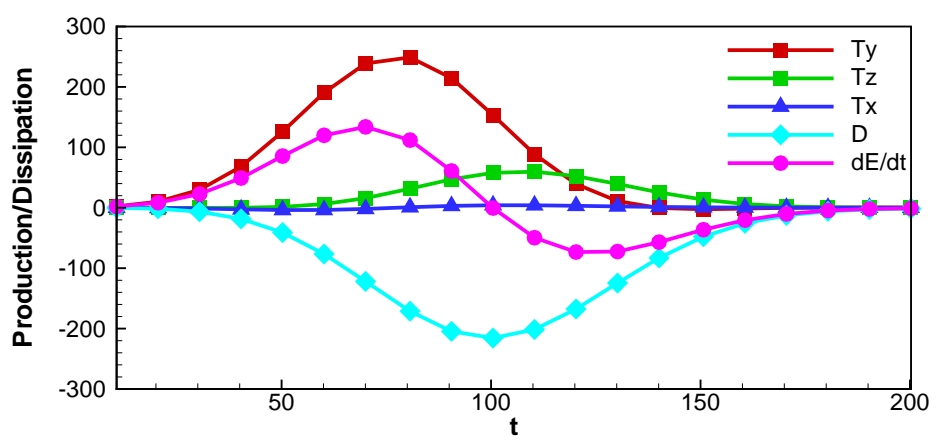

(a)

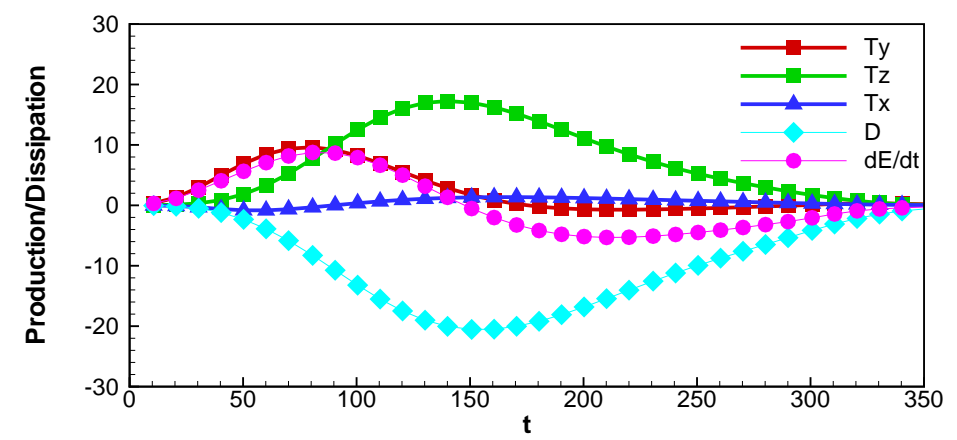

(b)

Figure 13. Perturbation kinetic energy time derivative, and production and dissipation terms versus time extracted by a linearized DNS initialized by the $\mathrm{VP}^{*}$ (a) and the $\mathrm{SP}^{*}(\mathrm{~b})$.

where the Cartesian tensor notation with the convention of summation over identical indices has been used for brevity, $u_{i}$ and $U_{i}$ being the perturbation and base flow velocity components, respectively, and $x_{i}$ being the spatial coordinates $x, y, z$. The two terms on the right hand side of equation (3.1) represent the exchange of energy with the base flow and the energy dissipation due to viscous effects, respectively. Since the largest component of the base flow is the streamwise one, the main production terms would be those with densities $T_{x}=-u u \frac{\partial U}{\partial x}, T_{y}=-u v \frac{\partial U}{\partial y}$, and $T_{z}=-u w \frac{\partial U}{\partial z}$. Figure 13 (a) and (b) show the time evolutions of these terms, integrated in the whole domain, along with the dissipation term and the variation of the kinetic energy, obtained by a linearized DNS initialized by $\mathrm{VP}^{*}$ and $\mathrm{SP}^{*}$, respectively. In both cases we have verified that the production terms associated with $V_{x, y, z}$ and $W_{x, y, z}$ are negligible with respect to $T_{x}$, $T_{y}$, and $T_{z}$. In the case of the VP, the production term associated with the wall-normal shear is the largest one. This term is always positive, in contrast to what is observed in the growth of varicose perturbations on a parallel streaky flow (compare with Fig. 6 (b) in Hoepffner et al. (2005)), where $T_{y}$ is the largest one at short times, but it is rapidly overtaken by the $T_{z}$ term. In both cases at very short times $(t<15$ in the case considered here) the Orr mechanism contributes to the growth of the $T_{y}$ term, but it gives a negative contribution for larger times $(t>15)$, when the perturbation is tilted downstream, so the main mechanism inducing a large energy growth should be a different one. Figure 13 (a) shows that $T_{y}$ peaks at $t \approx 80$, when the perturbation has been advected beyond the wake region, and enters in the quasi-parallel zone observed at $x>140$. This confirms that 


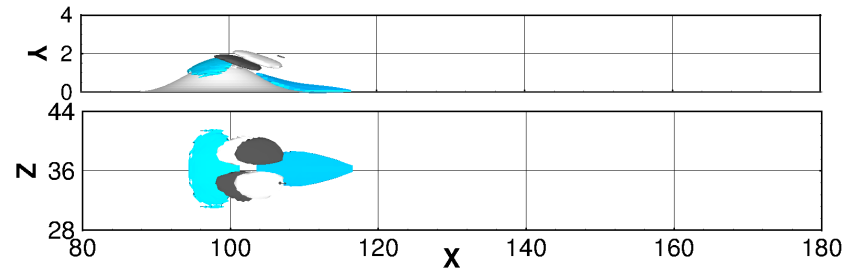

(a)

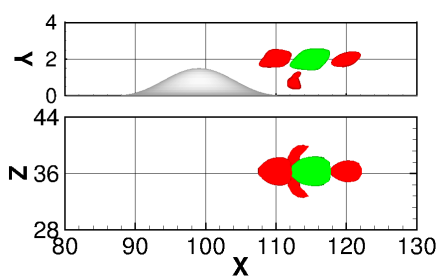

(b)

Figure 14. Base flow shear (grey, blue online), streamwise vorticity perturbation (white and black for $\omega_{x}= \pm 0.001$ ) at $t=0$ (a) and streamwise velocity perturbation (dark grey, red online, and light grey, green online, for $u^{\prime}= \pm 10^{-5}$ ) at $t=25$ (b) for the optimal perturbation obtained for $R e=300, k=1.5, T=50$.

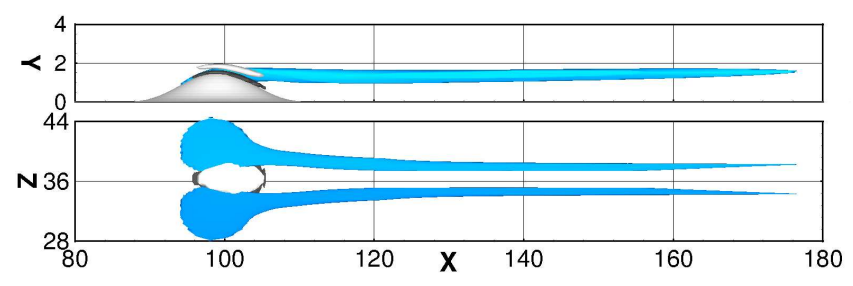

(a)

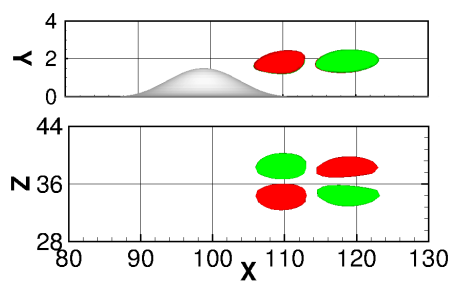

(b)

Figure 15. Base flow shear (grey, blue online), streamwise vorticity perturbation (white and black for $\omega_{x}= \pm 0.001$ ) at $t=0$ (a) and streamwise velocity perturbation (dark grey, red online, and light grey, green online, for $u^{\prime}= \pm 10^{-5}$ ) at $t=25$ (b) for the optimal perturbation obtained for $R e=300, k=1.5, T=200$.

the amplification mechanism for a varicose optimal perturbation is closely related to the wake past the bump. As shown in Fig. 14 (a), the alternated vortices (black and white) trasport the strong wall-normal shear (blue surfaces) which is localized in the wake region, creating patches of streamwise perturbation which grows in amplitude, shown in Fig. 14 (b). This mechanism also explains the large values of the streamwise velocity components observed in the optimal varicose perturbations for large bump heights. In fact, since the varicose perturbation should grow very fast to exploit the wall-normal shear of the wake, large initial values of $u$ would allow a rapid growth of the $T_{y}$ production term without waiting for $u$ to reach finite values as a result of the transport of the base flow streamwise velocity.

Concerning the SP, the amplification values are remarkably smaller (compare Figure 13 (a) and (b)), and the amplification mechanism is very different, being mainly related to the production term $T_{z}$. As one can observe in Figure $13(\mathrm{~b})$, the $T_{y}$ term is the largest one at very short times, due to the combined effect of the Orr mechanism and a small amount of transport of the low-momentum wake flow upwards in the boundary layer. However, such an amplifying effect is rapidly overtaken by the influence of the production term $T_{z}$, which grows in time up to $t \approx 150$ (consistent with the typical time at which the optimal perturbation drift from a varicose towards a sinuous shape). As shown in Figure 15 (a), the initial vortices (black and white), alternated in the wall-normal direction, transport the streamwise-elongated spanwise shear of the base flow (blue surfaces). This induces the formation, on the flanks of the initial vortices, of patches of streamwise disturbance with a growing amplitude, shown in Figure 15 (b). Being the spanwise base flow shear more extended in the streamwise direction, since it has its maximum in the interaction zone between streaks, the transient instability has more time to develop with respect to 
Transient growth in the flow past a three-dimensional smooth roughness element 21

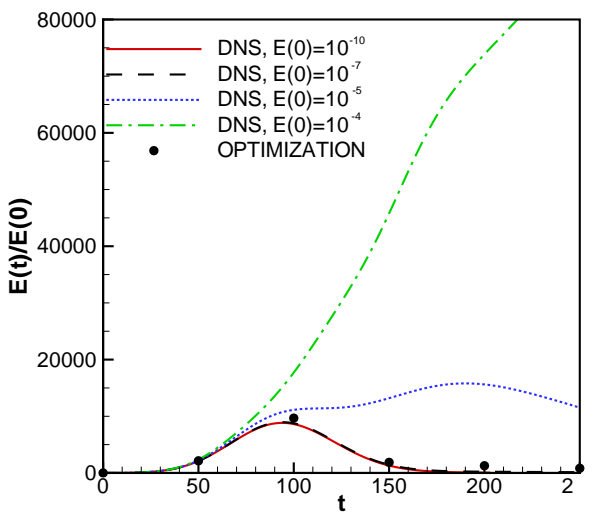

(a)

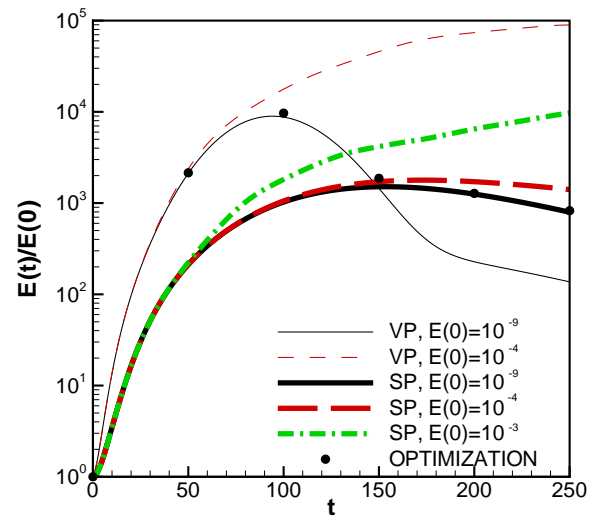

(b)

FIGURE 16. Energy gain versus time extracted by a DNS initialized by the optimal perturbation obtained for $R e=300, k=1.5, T=50$ ( $\mathrm{VP}^{*}$, left frame) and $T=200$ (SP*, right frame) with the initial energies indicated within the figure. The black dots indicate the optimal energy gain obtained at target time by the linear optimization.

the varicose case. This also explains why the streamwise perturbation at initial time is smaller than in the varicose case, since it has more time to grow, induced by the initial vortices. It is worth to notice that the evolution of the $T_{z}$ term for the sinuous case is similar to the one obtained by an optimal disturbance of a parallel streaky flow (compare with Fig. 6 (a) in Hoepffner et al. (2005)). However, some differences are observed: in the present case, the $T_{y}$ term achieves half of the $T_{z}$ peak values at $t=75$, wherease, in the parallel flow case (Hoepffner et al. 2005), its maximum values is only one tenth of the $T_{z}$ peak. As discussed before, such large initial values of $T_{y}$ are linked to the presence of a large wall-normall shear in the wake region.

\subsubsection{The route to transition}

In the above section we have identified the main terms inducing energy growth in the case of varicose and sinuous optimal disturbances, for the base flow at $R e=300$ and $k=1.5$. We will now analyze how this linear mechanisms can induce transition. Thus, we use the $\mathrm{VP}^{*}$ and $\mathrm{SP}^{*}$ to initialize several DNSs and we increase their energy in order to achieve transition. For the VP, Figure 16 (a) shows that the energy gain follows the linear behaviour up to $E(0)=10^{-5}$, when the energy gain begins to increase with respect to the linear case, but transition is still not observed. For larger initial energies, transition is triggered, as verified by computing the skin friction coefficient downstream of the bump. Concerning the SP, since the linear energy growth is smaller than that induced by the $\mathrm{VP}$, transition is observed for larger values of the initial energy. In particular, as shown in Figure $16(\mathrm{~b})$, an initial energy of order $E(0)=10^{-3}$ is needed to induce transition, which is one order of magnitude larger than that needed by the VP. Considering that both perturbations are localized on the bump and show a similar extention in all of the directions, a larger amplitude of the velocity components is needed for the SP to induce transition. This indicates that the VP are much more dangerous for the considered flow.

Figure 17 shows the contours of the instantaneous streamwise velocity component and of the streamwise vorticity perturbation extracted from the DNS initialized by the VP with initial energy $E_{0}=0.001$. The first two frames (at $t=0$ and $t=25$ ) show the tilting due to the Orr mechanism; then the vortices continue to increase in amplitude and start to change their initial shape and inclination. This is due to the fact that the 

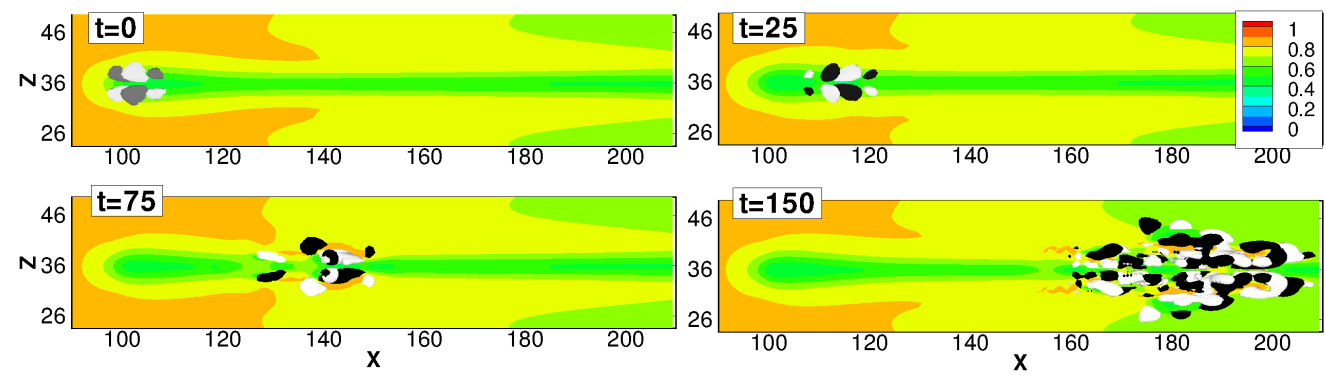

Figure 17. Contours of the instantaneous streamwise velocity at $y=2$ and surfaces of the streamwise vorticity perturbation (white and black) extracted by a DNS initialized by the optimal perturbation obtained for $R e=300, k=1.5, T=50$ with initial energy $E_{0}=0.001$ at $t=0$ (surfaces for $\omega_{x}= \pm 0.005$, first frame), $t=25$ (surfaces for $\omega_{x}= \pm 0.03$, second frame), $t=75$ and $t=150$ (surfaces for $\omega_{x}= \pm 0.08$, third and fourth frames, respectively).
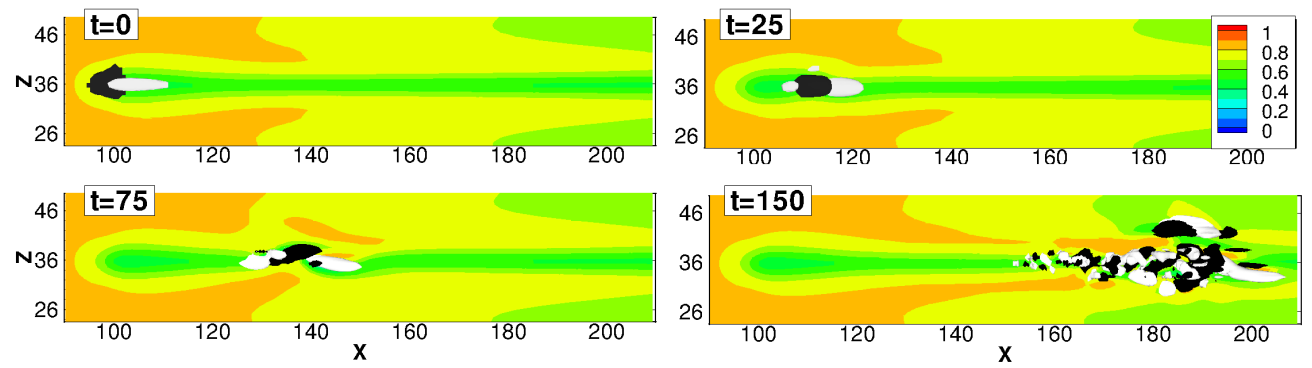

FiguRE 18. Contours of the instantaneous streamwise velocity at $y=2$ and surfaces of the streamwise vorticity perturbation (white and black) extracted by a DNS initialized by the optimal perturbation obtained for $R e=300, k=1.5, T=200$ with initial energy $E_{0}=0.01$ at $t=0, t=25$ (surfaces for $\omega_{x}= \pm 0.015$, first and second frame, respectively), $t=75$ and $t=150$ (surfaces for $\omega_{x}= \pm 0.1$, third and fourth frame, respectively).

streamwise disturbance induced by trasport of the mean flow reaches amplitudes which are comparable with the base flow, thus inducing modifications on the original streaks. Such base flow modifications, which are also responsible for the change of inclination of the vortices, can be observed in Figure 17 starting from the third frame. Since the streamwise perturbation has a varicose symmetry, the base flow streaks experience varicose oscillation (see the narrowing and enlargment of the streaks in the third frame of Figure 17, around $x \approx 140$ ). At $t=150$ (fourth frame) the streaks are broken down, and the vortices are split up in smaller ones. For $t>150$ the transitional wave packet is advected downstream, increasing its size in the spanwise and streamwise direction.

A different behaviour is observed during the evolution of the optimal SP, having initial energy $E_{0}=0.01$. The first two frames of Figure 18 show that the initial vortices are tilted in the streamwise direction and advected downstream. Then, the vortices begin to show a finite spanwise inclination with respect to the streamwise direction, creating inclined patches of streamwise disturbance that induce modifications of the base flow. In particular, the streaks present sinuous oscillations, which are clearly visible in the third frame of the Fig. 18, for $t=75$. At $t=150$ (fourth frame) the vortices are broken into smaller patches of vorticity, and the streaks have experienced breakdown. Then, the transitional wave packet is advected downstream, increasing its size in the spanwise and streamwise direction.

The two scenarios of transition observed here reflect the main features of the varicose 


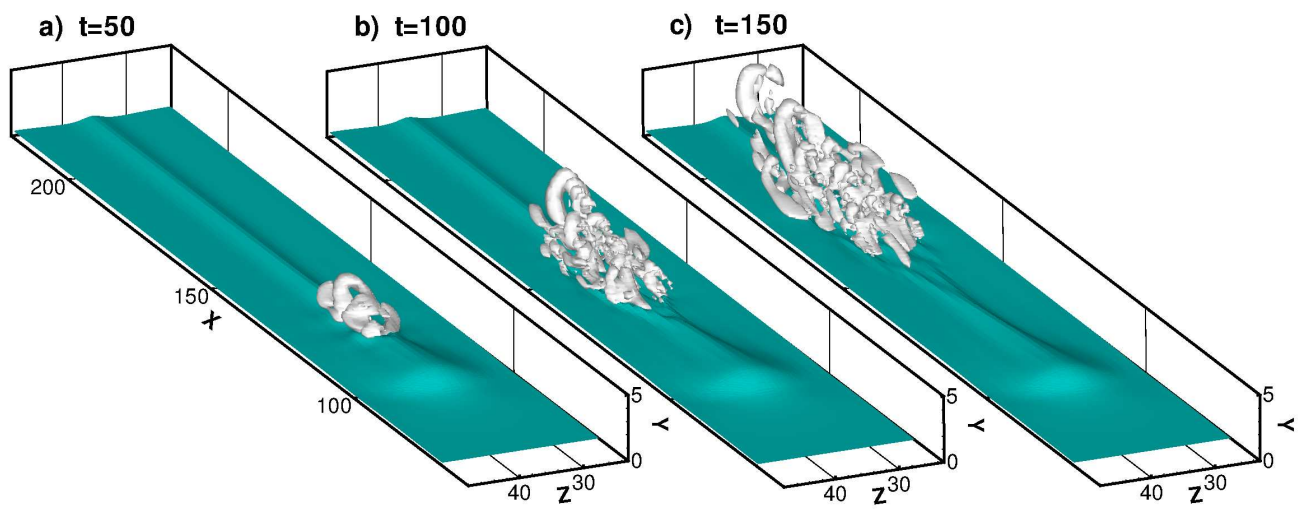

Figure 19. Surface of the instantaneous streamwise velocity $u=-0.2$ and of the Q-criterion (light gray for $Q=100$ ) extracted by a DNS initialized by the optimal perturbation obtained for $R e=300, k=1.5, T=50$ with initial energy $E_{0}=0.001$ at $t=50(\mathrm{a}), t=100$ (b), $t=150$ (c.)

and sinuous transition mechanisms for a parallel streaky flow, although in this case the most amplified perturbations are the varicose, not the sinuous ones. The main vortical structures recovered in the flow are shown in Figure 19 (a), (b), and (c) for VP*. The time sequence shows the generation of a small hairpin vortices at $t=50$, which is typically associated with streaks oscillations of varicose type. Such a vortical structure grows in size at $t=100$ and $t=150$, maintaing its main structure and further generating a train of hairpin vortices (Adrian 2007), which are often observed past roughness elements (see Acalar \& Smith (1987)). Concerning the evolution of SP*, no hairpin structure is observed. This confirms the different dynamics between the two transition scenarios and points out the importance of varicose optimal disturbances in a streaky flow induced by a smooth roughness element.

\section{Summary}

This work aims at describing the three-dimensional instability mechanisms which may lead the flow over a smooth roughness element to a rapid transition, by-passing the twodimensional asymptotical growth of TollmienSchlichting waves. This kind of roughness elements, which may resemble the imperfections of a flat surface, induce mild variations on the boundary-layer flow; therefore, the mechanism leading to transition could most likely be based on transient growth, like that recovered in a Blasius flow. Thus, we have performed a linear three-dimensional energy optimization analysis, looking for perturbations inducing the largest energy growth at a finite time in a boundary-layer flow in the presence of roughness elements of smooth axisymmetric shape. To this purpose, we have coupled the immersed boundary technique, useful to describe complex geometries, with a Lagrangian optimization in a three-dimensional framework. Smooth roughness elements having the shape of an axisymmetric three-dimensional bump have been studied, characterized by different heights, $k$ (non-dimensionalized with respect to the Blasius displacement thickness at the center of the roughness element, $\delta^{*}$ ). The influence of the Reynolds number has also been analyzed. We found that, for small bumps having $k<1$, the deformation of the base flow is weak, so that only negligible differences in the energy gain are observed with respect to the Blasius flow. In this case, the optimal perturbation 
has the shape of a wave packet with alternated quasi-streamwise vortices, similar to the one observed in a Blasius boundary layer, but localized in the streamwise and spanwise direction. For larger bumps $(k \geqslant 1)$ and small target times, we found that the optimal energy gain overtakes the one obtained for a Blasius flow up to several orders of magnitude. This indicates that, under certain conditions of environmental noise, this kind of bump may have a destabilizing effect on a short time scale due to non-normal transient growth, even if it can stabilize the TS waves on a larger time scale (see Piot et al. (2008)) in a low-noise environment. The optimal perturbations are strongly localized over the bump, and are characterized by initial vortices alternated in the wall-normal, or in the spanwise direction, with a sinuous or varicose symmetry, respectively. In particular, for large bump heights and Reynolds numbers, it appears that the optimal disturbances obtained for large target times $(t>150)$ are sinuous perturbation, whereas for smaller target times $(t \leqslant 150)$ they are characterized by a varicose structure. Moreover, the highest value of the energy gain is obtained for a varicose perturbation; in particular, we observed that varicose perturbations are able to induce an energy amplification up to 60 times larger than the sinuous ones at the same target time, pointing out the strong destabilizing effect of varicose instabilities for a streaky flow induced by a bump. The difference in the symmetry obtained for different target times can be explained by observing that the base flow itself is characterized by a strong shear having a varicose symmetry in the near field downstream of the bump, whereas, at larger abscissae, it turns into a nearlyparallel streaky flow. Thus, at small target times the optimal disturbances are strongly affected by such a varicose structure of the base flow, whereas they turn into sinuous perturbations when they reach the nearly parallel streaky flow far from the bump. Such different perturbations lead to different instability mechanisms creating hairpin vortices in the varicose case and oscillating streaks in the sinuous case. In particular, the varicose optimal perturbations are found to induce transition at smaller amplitudes and energies, meaning that they are indeed the most dangerous perturbations in a streaky flow induced by a bump. These results confirm the importance of taking into account the three-dimensionality of the base flow using three-dimensional global instability methods, in order to better understand the most likely scenarios of transition in a flow past a roughness element. Using global instability analysis will be even more crucial when dealing with non-smooth roughness elements with non-small aspect ratio, inducing stronger distortions of the boundary-layer base flow. In fact, for non-smooth roughness element such as cylinders, experimental observations (Tani et al. (1962); White (2002); White \& Ergin (2003); Fransson et al. (2004); Choudhari \& Fischer (2005)) have shown that the flow experiences transition at a given roughness Reynolds number, indicating the onset of a modal global instability linked to the three-dimensionality of the base flow. However, such a modal instability will have a different origin from the non-modal amplification mechanisms investigated here. Thus, the conclusions of the present study cannot be generalized to any roughness element shape and height, leaving the analysis of non-smooth roughness elements such as cylindrical ones to a future work.

\section{Acknowledgments}

Some computations have been performed on the Power 6 of the IDRIS, France.

\section{Appendix A. Immersed-boundary technique}

The IB technique is employed to take into account the presence of a single or multiple bodies inside the computational domain, while maintaining the use of Cartesian grids 
that do not need to conform to the geometry surfaces. Being the volume grid (and therefore its resolution at the wall) independent of the geometry surface discretization, the surface description focus uniquely on resolving the geometry. In this work, the body surface is discretized by means of unconnected triangles of size inversely proportional to the local curvature of the original surface (STL format), with the only requirement that the object must be a closed manifold. The body surface is overlapped onto the volume mesh, splitting it in fluid and solid regions, $\Gamma_{1}$ and $\Gamma_{2}$ (see figure 1 ). Note that the gray surface indicated in figure 1 is not a closed geometry. Indeed, in our implementation of the method, when the body intersects the boundaries of the computational domain, the domain boundary faces act as closure faces for the body. In order to assign the computational cells to each zone, a tagging procedure is performed, finding their relative position with respect to the body. This is done automatically by using a very efficient ray-tracing technique (O'Rourke (1993)), which consists in casting rays in one Cartesian direction, spanning from a reference fluid point and counting the intersections with the body surface. The number of valid intersections will then classify the cells as fluid (even number of intersections) or solid (odd number of intersections). With reference to figure 1 , in this work the rays are cast in the flow direction, starting for each point laying on the inlet surface. After the tagging, the fluid points that have at least one neighbor that is tagged as solid, are tagged as interface points.

At every point of the computational domain $\Gamma_{1}+\Gamma_{2}$, the governing equations (2.1) and (2.2) are solved. In the present work, the direct forcing of Mohd-Yusof (1997) is employed; therefore, at interface points a correction on the velocity value is forced at each time step so as to impose the desired velocity value on the body surface. Following Fadlun et al. (2000), the velocity at the interface points is obtained by linearly interpolating the velocity at a second grid point (which is obtained by directly solving the NavierStokes equations) and the velocity at the body surface, which conceptually corresponds to applying the momentum forcing inside the flow field. For each interface point, the direction towards the second grid point, i.e., the interpolation direction, is the Cartesian direction in which the distance with respect to the body is minimum. This also identifies the body intersections. Concerning the solid points, the zero-velocity forcing is applied (stationary boundary). It is worth noting that, as checked by Fadlun et al. (2000), for stationary boundary problems, different treatments inside the solid body do not affect the external flow. The present IB technique is coupled with the Lagrangian optimization described in the following.

\section{Appendix B. Direct-adjoint optimization technique}

The Lagrange multiplier technique consists in seeking extrema of the augmented functional $\mathcal{L}$, provided in equation $(2.4)$ with respect to every independent variable. Integrating by parts and setting to zero the first variation of $\mathcal{L}$ with respect to $u^{\prime}, v^{\prime}, w^{\prime}, p^{\prime}$ leads to the adjoint equations:

$$
\begin{gathered}
b_{t}=-b_{x} U-(b V)_{y}+c V_{x}-(b W)_{z}+W_{x} d-a_{x}-\frac{b_{x x}+b_{y y}+b_{z z}}{R e} \\
c_{t}=-(c U)_{x}-c_{y} V+b U_{y}-(c W)_{z}+W_{y} d-a_{y}-\frac{c_{x x}+c_{y y}+c_{z z}}{R e} \\
d_{t}=-(d U)_{x}-(d V)_{y}+U_{z} b+V_{z} c-W d_{z}-a_{z}-\frac{d_{x x}+d_{y y}+d_{z z}}{R e} \\
b_{x}+c_{y}+d_{z}=0
\end{gathered}
$$


where $\mathbf{q}^{\dagger}=(a, b, c, d)^{T}$ is now identified as the adjoint vector. By using the boundary conditions of the direct problem, one obtains:

$$
\begin{gathered}
b=0, c=0, d=0, \text { for } \quad y=y_{w} \quad \text { and } \quad y=L_{y} \\
b=0, c=0, d=0, \text { for } \quad x=x_{\text {in }} \quad \text { and } \quad x=x_{\text {out }}
\end{gathered}
$$

where the zero perturbation condition at the wall is imposed by means of the immersed boundary technique for both the direct and adjoint equations; it is noteworthy that, using the direct forcing approach, no explicit forcing term appears in the adjoint equations. Nullifying the terms at $t=T$ one obtains the compatibility conditions (Zuccher et al. 2004):

$$
\frac{2 u^{\prime}}{E(0)}-b=0, \frac{2 v^{\prime}}{E(0)}-c=0, \frac{2 w^{\prime}}{E(0)}-d=0, \text { for } \quad t=T
$$

By solving the direct and adjoint equations at each step of the iterative procedure, the first variation of the augmented functional with respect to $\mathbf{q}$ and $\mathbf{q}^{\dagger}$ is set to zero. Moreover, the gradient of $\mathcal{L}$ with respect to the initial state $\mathbf{q}_{0}$ has to vanish within a reasonable number of iterations. In order to achieve convergence efficiently, a conjugate gradient algorithm is used. The initial state is updated in the steepest ascent direction, denoted as:

$$
\nabla_{\mathbf{q}_{0}} \mathcal{L}=-2 \mathbf{q}_{0} \frac{E(T)}{E(0)^{2}}+\mathbf{q}^{\dagger}(0)
$$

with an adjustable step length $\alpha$, so that $\mathbf{q}_{0}^{(n+1)}=\mathbf{q}_{0}^{n}+\alpha^{n} \nabla_{\mathbf{q}_{0}} \mathcal{L}^{n}$. After the first iteration in the steepest ascent direction, the successive steps are taken along a conjugate direction, $\Lambda \mathbf{q}_{0}$, which is computed on the basis of the gradient at two consecutive iterations according to $\Lambda \mathbf{q}_{0}^{(n+1)}=\nabla_{\mathbf{q}_{0}} \mathcal{L}^{(n+1)}+\beta^{(n+1)} \Lambda \mathbf{q}_{0}^{n}$. The value of the parameter $\beta^{(n+1)}$ is computed by means of the Polak-Ribière formula (Polak \& Ribière 1969). The step length $\alpha$ has been chosen small enough in order to ensure convergence to the optimal value.

The optimization procedure for a chosen target time $T$ can be summarized as follows:

(a) An initial guess is taken for the initial condition, $q_{0}$, at $t=0$, with an associated initial energy $E(0)$.

(b) The direct problem is integrated from $t=0$ to $t=T$.

(c) At $t=T$, the initial state for the adjoint problem is provided by the compatibility condition (B 3).

(d) The adjoint problem (B 1) is integrated backward in time from $t=T$ to $t=0$, starting from the initial state of step $(c)$.

(e) At $t=0$, the initial direct state is updated in the direction of the conjugate gradient with step length $\alpha$ and $\beta$ computed according to the Polak-Ribière formula $(\beta=0$ is imposed at the first iteration).

$(f)$ The objective function $E(T) / E(0)$ is evaluated; if its increase between two successive iterations is smaller than a chosen threshold, $\epsilon=10^{-5}$, the loop is stopped, otherwise the procedure is continued from step $(b)$.

The performance of the optimization procedure, as well as a detailed convergence study for the case of the Blasius boundary-layer flow, are provided in Cherubini et al. $(2010 b, a)$.

\section{REFERENCES}

ACAlaR, M. \& Smith, C. 1987 A study of hairpin vortices in a laminar boundary layer: part 1, hairpin vortices generated by a hemisphere protuberance . J. Fluid Mech. 175, 1-41.

Adrian, R. J. 2007 Hairpin vortex organization in wall turbulence. Phys. Fluids 19, 041301. 
Andersson, P., Brandt, L., Bottaro, A. \& Henningson, D. S. 2001 On the breakdown of boundary layer streaks. J. Fluid Mech. 428, 29-60.

Barkley, D., Gomes, G. M. \& Henderson, R. D. 2002 Three-dimensional instability in flow over a backward-facing step. J. Fluid Mech. 473, 167-190.

BArkley, D. \& Henderson, R. D. 1996 Floquet stability analysis of the periodic wake of a circular cylinder. J. Fluid Mech 322 (215-241).

Blackburn, H.M., Barkley, D. \& Sherwin, S.J. 2008 Convective instability and transient growth in flow over a backward-facing step. J. Fluid Mech. 603 (271-304).

Bottaro, A. 1990 Note on open boundary conditions for elliptic flows . Num. Heat Transfer B 18, 243-256.

Brandt, L., Schlatter, P. \& Henningson, D. S. 2004 Transition in a boundary layers subject to free-stream turbulence. J. Fluid Mech. 517, 167-198.

Butler, K. M. \& FArrell, B. F. 1992 Three-dimensional optimal perturbations in viscous shear flow. Phys. Fluids A 4, 1637-1650.

Cherubini, S., De Palma, P., Robinet, J.-Ch. \& Bottaro, A. $2010 a$ Rapid path to transition via nonlinear localized optimal perturbations . Phys. Rev. E 82, 066302.

Cherubini, S., De Palma, P., Robinet, J.-Ch. \& Bottaro, A. 2011 The minimal-seed of turbulent transition in a boundary layer . J. Fluid Mech. 689, 221-253.

Cherubini, S., Robinet, J.-Ch., Bottaro, A. \& De Palma, P. $2010 b$ Optimal wave packets in a boundary layer and initial phases of a turbulent spot . J. Fluid Mech. 656, 231-259.

Cherubini, S., Robinet, J.-C. \& De Palma, P. 2010c The effects of non-normality and non-linearity of the Navier-Stokes operator on the dynamics of a large laminar separation bubble. Phys. of Fluids 22 (1), 014102.

Choudhari, M. \& Fischer, P. 2005 Roughness-induced transient growth: nonlinear effects . 35th AIAA Fluid Dynamics Conference and Exhibit, Toronto, Ontario Canada AIAA2005-4765.

Corbett, P. \& Bottaro, A. 2000 Optimal perturbations for boundary layers subject to stream-wise pressure gradient . Phys. Fluids 12, 120-130.

Fadlun, E. A, Verzicco, R., Orlandi, P. \& Mohd-Yusof, J. 2000 Combined immersedboundary finite-difference methods for three-dimensional complex flow simulations . $J$. Comput. Phys. 161, 35.

FArrell, B. 1988 Optimal excitation of perturbations in viscous shear flow . Phys. Fluids 31, $2093-2102$.

Fransson, J.H.M., Brandt, L., Talamelli, A. \& Cossu, C. 2004 Experimental and theoretical investigation of the nonmodal growth of steady streaks in a flat plate boundary layer. Phys. Fluids 16 (10), 3627-3638.

Fransson, J.H.M., Brandt, L., Talamelli, A. \& Cossu, C. 2005 Experimental study of the stabilization of TollmienSchlichting waves by finite amplitude streaks. Phys. Fluids 17 (5), 054110.

Fransson, J.H.M., Talamelli, A., Brandt, L. \& Cossu, C. 2006 Delaying transition to turbulence by a passive mechanism . Phys. Rev. Lett. 96 (6), 064501.

Gallaire, F., Marquillie, M. \& Ehrenstein, U. 2007 Three-dimensional transverse instabilities in detached boundary-layers . J. Fluid Mech. 571, 221-233.

Gaster, M., Grosch, C. E. \& Jackson, T. L. 1994 The velocity field created by a shallow bump in a boundary layer. Phys. Fluids 6, 3079.

Giannetti, F. \& Luchini, P. 2007 Structural sensitivity of the first instability of the cylinder wake. J. Fluid Mech. 581, 167-197.

Gregory, N. \& WALKER, W. S. 1956 The effect on transition of isolated surface excrescences in the boundary layer. R. \& M. No. 2779, A.R.C. Technical Report.

Hoepffiner, J., Brandt, L. \& Henningson, D. S. 2005 Transient growth on boundary layer streaks. J. Fluid Mech. 537, 91-100.

Joslin, R. D. \& Grosch, C. E. 1995 Growth characteristics downstream of a shallow bump: Computation and experiment. . Phys. Fluids 7 (12), 3042-3047.

Lipatov, I.I. \& Vinogradov, I.V. 2000 Three-dimensional flow near surface distortions for the compensation regime. Philos. Trans. R. Soc. London A 358, 3143-3153.

LuChini, P. 2000 Reynolds number indipendent instability of the Blasius boundary layer over a flat surface: optimal perturbations . J. Fluid Mech. 404, 289-309. 
Marquet, O., Sipp, D., Chomaz, J.-M. \& Jacquin, L. 2008 Amplifier and resonator dynamics of a low-Reynolds-number recirculation bubble in a global framework. J. Fluid Mech.605, 429-443.

Mohd-Yusof, J. 1997 Combined immersed boundaries/B-splines methods for simulations of flows in complex geometries. CTR Annual Research Briefs, NASA Ames/Stanford University.

Monokrousos, A., Akervik, E., Brandt, L. \& Henningson, D. S. 2010 Global threedimensional optimal disturbances in the Blasius boundary-layer flow using time-steppers . J. Fluid Mech. 650, 181-214.

O'Rourke, J. 1993 Computational Geometry in C . Cambridge Univ. Press.

OrR, W. MF. 1907 The stability or instability of the steady motions of a liquid. Part I . Proceedings of the Royal Irish Academy, A 27, 9-68.

Piot, E., Casalis, G. \& Rist, U. 2008 Stability of the laminar boundary layer flow encountering a row of roughness elements: Biglobal stability approach and dns. European Journal of Mechanics - B/Fluids 27 (6), $684-706$.

Polak, E. \& Ribière, G. 1969 Note sur la convergence de directions conjugées. Rev. Francaise Informat Recherche Operationnelle 16, 35-43.

Schmid, P. \& Henningson, D. 2001 Stability and transition in shear fows. Springer.

Schmid, P. J. 2000 Linear stability theory and by pass transition in shear flows . Phys. Plasmas 7, 1788-1794.

Schoppa, W. \& Hussain, F. 2002 Coherent structure generation in near-wall turbulence. J. Fluid Mech. 453, 57-108.

Tani, I., Komoda, H., Komatsu, Y. \& Iuchi, M. 1962 Boundary-layer transition by isolated roughness. Tech. Rep. 375 .

TheofiLis, V. 2003 Advances in global linear instability of nonparallel and three-dimensional flows. Prog. in Aerospace Sciences 39, 249-315.

Theofilis, V., Hein, S. \& Dallmann, U. 2000 On the origins of unsteadiness and three dimensionality in a laminar separation bubble. Phil. Trans. R. Soc. Lond. 358 (1777), 3229-3246.

Verzicco, R. \& Orlandi, P. 1996 A finite-difference scheme for the three-dimensional incompressible flows in cylindrical coordinates . J. Comp. Phys. 123 (2), 402-414.

White, E. B. 2002 Transient growth of stationary disturbances in a flat plate boundary layer . Phys. Fluids 14 (12), 4429.

White, E. B. \& Ergin, F. G. 2003 Receptivity and transient growth of roughness-induced disturbances. AIAA-2003-4243.

Zuccher, S., Luchini, P. \& Bottaro, A. 2004 Algebraic growth in a blasius boundary layer: optimal and robust control by mean suction in the nonlinear regime. Eur. J. Mech. B/Fluids 513, 135-160. 